\title{
First-principles calculations of the high- temperature phase transformation in yttrium tantalate
}

\section{Citation}

Feng, Jing, Samuel Shian, Bing Xiao, and David R. Clarke. 2014. "First-Principles Calculations of the High-Temperature Phase Transformation in Yttrium Tantalate." Physics Review B 90, no. 9: 094102.

\section{Published Version}

doi:10.1103/PhysRevB.90.094102

\section{Permanent link}

http://nrs.harvard.edu/urn-3:HUL.InstRepos:12965656

\section{Terms of Use}

This article was downloaded from Harvard University's DASH repository, and is made available under the terms and conditions applicable to Open Access Policy Articles, as set forth at http:// nrs.harvard.edu/urn-3:HUL.InstRepos:dash.current.terms-of-use\#OAP

\section{Share Your Story}

The Harvard community has made this article openly available.

Please share how this access benefits you. Submit a story.

\section{Accessibility}




\title{
FIRST-PRINCIPLES CALCULATIONS OF THE HIGH-TEMPERATURE PHASE TRANSITION IN YTTRIUM TANTALATE
}

\author{
Jing Feng ${ }^{1}$, Samuel Shian ${ }^{1}$, Bing Xiao $^{2}$ and David R. Clarke ${ }^{{ }^{*}}$ \\ 1 School of Engineering and Applied Sciences, Harvard University, Cambridge, MA 02138, US \\ 2 Departments of Physics, College of Science and Engineering, Temple University, Philadelphia, \\ PA, 19122, USA
}

\section{ABSTRACT}

The high temperature phase transition between the tetragonal (scheelite) and monoclinic (fergusonite) forms of yttrium tantalite $\left(\mathrm{YTaO}_{4}\right)$ has been studied using a combination of firstprinciples calculations and a Landau free-energy expansion. Calculations of the Gibbs free energies show that the monoclinic phase is stable at room temperature and transforms to the tetragonal phase at $1430{ }^{\circ} \mathrm{C}$, close to the experimental value of $1426 \pm 7{ }^{\circ} \mathrm{C}$. Analysis of the phonon modes as a function of temperature indicate that the transformation is driven by softening of transverse acoustic modes with symmetry $E_{u}$ in the Brillouin zone center rather than the Raman-active $\mathrm{B}_{\mathrm{g}}$ mode. Landau free energy expansions demonstrate that the transition is second-order and, based on the fitting to experimental and calculated lattice parameters; it is found that the transition is a proper rather than a pseudo-proper type. Together, these findings are consistent with the transition being ferroelastic. 


\section{INTRODUCTION}

A number of compounds are now known to undergo a ferroelastic phase $\operatorname{transition}^{1-5}$ on cooling or in response to an applied stress. They share the characteristics of being second order with a continuous change in the unit cell volume at the transition. Furthermore, group theory analysis indicates that the high and low symmetry phases are connected by an irreducible representation in the Brillouin zone center. In addition, an intriguing feature of materials that can undergo a ferroelastic phase transition is that they are capable of exhibiting toughening if the stress is sufficiently high to trigger the transformation from one variant to another ${ }^{6,7}$. This occurs, for instance, in yttria-stabilized zirconia ${ }^{7-10}$. In this oxide material, the high-temperature cubic phase undergoes a ferroelastic transformation to crystallographically equivalent tetragonal variants, which can be switched by an applied stress, dissipating energy and thereby producing toughening. This is believed to be the basis for the unusually high fracture toughness of YSZ thermal barrier coating at elevated temperatures ${ }^{7}$. In the search for other oxides capable of ferroelastic toughening, we have been studying the transformations in $\mathrm{YTaO}_{4}$ to determine whether it, too, exhibits a ferroelastic phase transition. From in-situ X-ray diffraction experiments, the high symmetry scheelite- $\mathrm{YTaO}_{4} \quad\left(\mathrm{I}_{41} / \mathrm{a}\right)$ is stabilized over monoclinic fergusonite- $\mathrm{YTaO}_{4}\left(\mathrm{C}_{2} / \mathrm{c}\right)$ phase above $1426 \pm 7{ }^{\circ} \mathrm{C}$. However, the detailed transition mechanism in $\mathrm{YTaO}_{4}$ system has not been established and this motivates the first-principles calculations presented here.

One of the earliest studies to understand the mechanism underlying ferroelastic phase transitions using first-principles methods was reported by Carpenter and coworkers in the crystalline silica $\left(\mathrm{SiO}_{2}\right)^{11}$. The tetragonal stishovite $\left(\mathrm{P}_{42} / \mathrm{mmm}\right)$ to orthorhombic $\mathrm{CaCl}_{2}$ type $\mathrm{SiO}_{2}$ 
(Pnnm) is rationalized as a prototypical pseudo-proper ferroelastic transition under pressure. Both experiments and theoretical calculations confirmed that the transition is driven by the softening of the zone-center $\mathrm{B}_{1 \mathrm{~g}}$ optical mode, a Raman-active mode in the tetragonal-stishovite phase. In addition, the coupling between $\mathrm{B}_{1 \mathrm{~g}}$ optical mode and the transverse acoustic modes results in non-linear variations of the elastic constants as a function of pressure. In Refs. ${ }^{11-14}$, expressions for the Landau free energy expansion for the high symmetry stishovite phase were successfully derived and applied to explain the observed spontaneously macroscopic strains and elastic behaviors. The transformation under pressure of another oxide, the $\mathrm{ScVO}_{4}$ compound was studied by Panchal et al. ${ }^{15}$ by Raman scattering and density-functional perturbation theory (DFPT) calculations. They found that the scheelite $\left(\mathrm{I}_{41} / \mathrm{a}\right)$-to-fergusonite $\left(\mathrm{I}_{2} / \mathrm{a}\right)$ phase transition in this oxide was a displacive second-order ferroelastic transition and occurred at $9 \mathrm{GPa}$. However, the softening of $\mathrm{B}_{1 \mathrm{~g}}$ mode in the scheelite phase, which was assumed to be the driving force for phase transition, was not detected by Raman scattering. There is also evidence that the ferroelastic phase transition can only be triggered by the softening of transverse acoustic modes in the Brillouin zone. For instance, Dove ${ }^{16}$ and collaborators found that the ferroelastic transitions in $(\mathrm{Na}, \mathrm{K}) \mathrm{AlSi}_{3} \mathrm{O}_{8}$ and $(\mathrm{Sr}, \mathrm{Ca}) \mathrm{Al}_{2} \mathrm{Si}_{2} \mathrm{O}_{8}$ feldspar solid solutions as a function of pressure are indeed driven by the vanishing of a combination of transverse acoustic elastic constants and without softening of any optical modes. Furthermore, other ferroelastic phase transitions, such as the temperature induced transitions in $\mathrm{Li}_{2} \mathrm{TiGeO}_{5}{ }^{17}$ and $\mathrm{CaCl}_{2}{ }^{18}$ and pressure induced transitions in $\mathrm{RuO}_{2}{ }^{19}$ and $\mathrm{SnO}_{2}{ }^{20}$, have been successfully studied by first principles calculations, to get the mechanism of the ferroelastic phase transitions. These studies indicate that the transformation is associated with a softening mode. 
Fig 1 shows the crystal structure of high temperature tetragonal (scheelite) and low temperature monoclinic (fergusonite) phases of $\mathrm{YTaO}_{4}$. At ambient conditions, $\mathrm{YTaO}_{4}$ exists in the fergusonite monoclinic structure (space group $\mathrm{I}_{12} / \mathrm{a} 1$, No. 15 , point group $\mathrm{C}_{2 \mathrm{~h}}^{6}$ ). With increasing the temperature, the phase changes to the scheelite tetragonal structure (space group $\mathrm{I}_{1} / \mathrm{a}$, No.88, point group $\mathrm{C}_{4 \mathrm{~h}}^{6}$ ). Both phases contain four formula units per crystal cell with $\mathrm{TaO}_{4}$ tetrahedra bonded to $\mathrm{Y}$ and $\mathrm{O}$ ions as shown. Crystallographically, the two structures are related by a $90^{\circ}$ rotation around the common a-axis, given by the matrix:

$$
\left(\begin{array}{l}
a_{m} \\
b_{m} \\
c_{m}
\end{array}\right)=\left(\begin{array}{ccc}
0 & 1 & -1 \\
0 & 1 & 0 \\
1 & 0 & 0
\end{array}\right)\left(\begin{array}{l}
a_{t} \\
b_{t} \\
c_{t}
\end{array}\right)
$$

In this orientation, the $\mathrm{C}_{2}$ rotational axis in the [001] direction of the tetragonal phase is parallel to $b$ direction in monoclinic phase.

In this work, we report the results of tetragonal-to-monoclinic phase transition in $\mathrm{YTaO}_{4}$ by combining the theoretical calculations with the high temperature X-ray diffraction measurements. The Landau free energy expansion for the tetragonal scheelite- $\mathrm{YTaO}_{4}$ has been derived assuming, initially, that it undergoes pseudo-proper transformation and the corresponding spontaneously macroscopic strains were obtained. Using the DFPT, the phonon spectra and the soft-modes were discussed. Our results indicate that the phase transition in $\mathrm{YTaO}_{4}$ system is driven by the softening of transverse acoustic modes and that the transformation is a proper type, rather than pseudo-proper type initially assumed. 


\section{LANDAU FREE-ENERGY EXPANSION}

In Landau theory, a second-order phase transition is described using group-theory, in which the excess free energy of the crystals is expanded as a function of order parameter, $Q$, near the transition temperature. The order parameter is zero in the high symmetry phase, and increases as this symmetry is progressively "broken" in the low symmetry phase. For a temperaturedependent phase transition, the order parameter is set as unity at absolute zero. The Landau free energy, $G^{\prime}$, is the difference in free energy between the low symmetry phase, $G$, and the high symmetry phase, $G_{o},{ }^{21}$. In ferroelastic crystals, the transformation introduces lattice distortions, which can be described using spontaneous strains. The elastic energy $G_{e}$ can be directly related to the changes in the lattice parameters, which are measureable. Furthermore in a pseudo-proper transformation, the spontaneous strains do not coincide with the order parameter, instead, they interacts through coupling coefficient $\lambda^{22}$ and contribute to the coupling free energy $G_{c}$. Therefore, the Landau free energy can be written as the sum of the three energies ${ }^{23-26}$ :

$$
G^{\prime}=G-G_{0}=G_{Q}+G_{e}+G_{c}
$$

where

$$
\begin{aligned}
& G_{Q}=\frac{1}{2} a^{\prime}\left(T-T_{C}\right) Q^{2}+\frac{1}{4} b^{\prime} Q^{4} \\
& G_{e}=\frac{1}{2} \sum_{i, j=1,3} c_{i j}^{0} e_{i} e_{j}+\frac{1}{2} \sum_{k=4,6} c_{k k}^{0} e_{k}^{2} \\
& G_{c}=\sum_{s} \lambda_{s} e_{s} Q+\sum_{n s} \lambda_{n s} e_{n s} Q^{2}
\end{aligned}
$$


The standard Landau expansion $G_{Q}$ is limited to $4^{\text {th }}$ order, where $a^{\prime}$ and $b^{\prime}$ are coefficients in the series expansion. The elastic energy $G_{e}$ is calculated from the strains and the elastic constants. The coupling energy $G_{c}$ is limited to the $2^{\text {nd }}$ order and since the symmetrybreaking strain are directly proportional to the order parameter, the sum of their exponents should also follow Landau's expansion (only two are used here). Thus, the coupling between the order parameter $Q$ and spontaneous strains $e$ can be categorized into symmetry-breaking strain (subscript s) and non-symmetry breaking strain (subscript ns) with bilinear and linear-quadratic couplings with the order parameter, respectively. Note that for the high symmetry phase, all the spontaneous strains and the $Q$ vanish and the Landau free energy is simply zero.

To expand the above equation for $\mathrm{YTaO}_{4}$, the relation between the tetragonal and the monoclinic lattice parameters must be formulated using an irreducible representation and then the spontaneous strains can be derived. The crystal symmetries of the high-temperature tetragonal and low temperature monoclinic phases of $\mathrm{YTaO}_{4}$ are reported to be $\left(\mathrm{I}_{41} / \mathrm{a}\right)$ and $\left(\mathrm{C}_{2} / \mathrm{c}\right)$ respectively ${ }^{27-29}$. In this transformation, the spontaneous strains can be expressed in terms of the lattice parameters of both the monoclinic and tetragonal phases using the following ${ }^{30}$

$$
e_{1}=\frac{c_{M} \sin \beta}{a_{T}}-1, e_{2}=\frac{a_{M}}{a_{T}}-1, e_{3}=\frac{b_{M}}{c_{T}}-1, e_{4}=e_{5}=0, e_{6}=-\frac{c_{M} \cos \beta}{2 a_{T}}
$$

Analysis of the possible lattice correspondences between these structures using the group theory ISOTROPY code [http://stokes.byu.edu/iso/isotropy.php] indicate that they can be expressed in terms of symmetry-breaking strain based on the $\mathrm{B}_{1 \mathrm{~g}}$ symmetry in the Brillioun zone. The symmetry-breaking strains are $e_{x x}-e_{y y}$ and $e_{x y}$ (or $e_{1}-e_{2}$ and $e_{6}$ in the Voigt notation) relative to the high-temperature tetragonal phase. They can be defined in terms of the crystallographic coordinates relative to a Cartesian coordinate system for the monoclinic to 
tetragonal phase transition shown in Fig. 2. These two strains are the symmetry adaptive strains which determine the low symmetry phase completely. The non-symmetry-breaking strains anticipated for the phase transition are $e_{1}+e_{2}$ and $e_{3}$ with $\mathrm{A}_{1 \mathrm{~g}}$ symmetry in $\mathrm{BZ}$ center. Note that the transformation does not depend on the volume strain $e_{1}+e_{2}+e_{3}$.

From these considerations, Eq. $2 \mathrm{a}-2 \mathrm{~d}$ can be expressed as follow:

$$
\begin{aligned}
& G^{\prime}\left(Q, e_{i}\right)=\frac{1}{2} a^{\prime}\left(T-T_{c}\right) Q^{2}+\frac{1}{4} b^{\prime} Q^{4}+\lambda_{1}\left(e_{1}+e_{2}\right) Q^{2}+\lambda_{2} e_{3} Q^{2}+\lambda_{3}\left(e_{1}-e_{2}\right) Q+\lambda_{4} e_{6} Q+ \\
& \frac{1}{4}\left(c_{11}^{0}+c_{12}^{0}\right)\left(e_{1}+e_{2}\right)^{2}+\frac{1}{4}\left(c_{11}^{0}-c_{12}^{0}\right)\left(e_{1}-e_{2}\right)^{2}+c_{13}^{0}\left(e_{1}+e_{2}\right) e_{3}+\frac{1}{2} c_{33}^{0} e_{3}^{2}+\frac{1}{2} c_{66}^{0} e_{6}^{2}
\end{aligned}
$$

The normal elastic constants involved in the phase transitions are given for monoclinic phase as $c_{11}^{0}, c_{12}^{0}, c_{13}^{0}, c_{33}^{0}$ and $c_{66}^{0}$. Exact relationships between the strains and $Q$ can be calculated by invoking equilibrium, i.e., the first derivative of the Landau free energy with respect to both the order parameter and spontaneous strains $\left(e_{1}-e_{2}, e_{1}+e_{2}\right.$, and $\left.e_{6}\right)$ are zero:

$$
\frac{\partial G^{\prime}}{\partial Q}=0, \quad \frac{\partial G^{\prime}}{\partial\left(e_{1}-e_{2}\right)}=0, \quad \frac{\partial G^{\prime}}{\partial\left(e_{1}+e_{2}\right)}=0, \quad \frac{\partial G^{\prime}}{\partial e_{6}}=0
$$

They are found to be:

$$
\begin{gathered}
e_{1}+e_{2}=\frac{2\left(\lambda_{2} c_{13}^{0}-\lambda_{1} c_{33}^{0}\right)}{c_{33}^{0}\left(c_{11}^{0}+c_{12}^{0}\right)-2 c_{13}^{0}{ }^{2}} Q^{2}, \\
e_{1}-e_{2}=-\frac{2 \lambda_{3}}{c_{11}^{0}-c_{12}^{0}} Q,
\end{gathered}
$$




$$
\begin{gathered}
e_{3}=\frac{2 \lambda_{1} c_{13}^{0}-\lambda_{2}\left(c_{11}^{0}+c_{12}^{0}\right)}{c_{33}^{0}\left(c_{11}^{0}+c_{12}^{0}\right)-2 c_{13}^{0}{ }^{2}} Q^{2}, \\
e_{6}=\frac{-\lambda_{4}}{c_{66}^{0}} Q,
\end{gathered}
$$

Later, using strains, defined in equations (3), and calculated from the first principles lattice parameters together with the computed elastic constants (Table 2), the coupling coefficients, $\lambda_{1}$, $\lambda_{2}, \lambda_{3}$ and $\lambda_{4}$, were obtained by solving equations (6) - (9).

In turn, the renormalized Landau excess free energy for the monoclinic $\mathrm{YTaO}_{4}$ phase can be written as

$$
\mathrm{G}^{\prime}=\frac{1}{2} a^{\prime}\left(T-T_{c}^{*}\right) Q^{2}+\frac{1}{4} b^{*} Q^{4}
$$

with the renormalized transition temperature $T_{c}^{*}$ and coefficient $b^{*}$ being

$$
\begin{gathered}
T_{c}^{*}=T_{c}+\frac{\lambda_{3}^{2}}{\left(c_{11}^{0}-c_{12}^{0}\right) / 2}+\frac{\lambda_{4}^{2}}{c_{66}^{0}}, \\
b^{*}=b^{`}-2 \frac{\left.2 \lambda_{1}^{2} c_{33}^{0}+\lambda_{3}^{2}\left(c_{11}^{0}+c_{12}^{0}\right)-4 \lambda_{1} \lambda_{2} c_{13}^{0}\right)}{c_{33}^{0}\left(c_{11}^{0}+c_{12}^{0}\right)-2 c_{13}^{0}},
\end{gathered}
$$

Consequently, the temperature dependence of the order parameter will be given by

$$
Q^{2}=\frac{a^{\prime}}{b^{*}}\left(T_{c}^{*}-T\right), \quad T<T_{c}^{*},
$$

This has the familiar form of a second order, mean-field transition, with the order parameter dependent of the square root of reduced temperature. However, unlike the tetragonal-toorthorhombic ferroelastic phase transition in $\mathrm{SiO}_{2}$ system ${ }^{14}$, for instance, the transition in the $\mathrm{YTaO}_{4}$ system involves an additional dependence on the symmetry-breaking strain $e_{6}$. 
Specifically, the renormalized transition temperature, equation 10 , is dependent on $e_{6}$, whereas the constant $b^{*}$ is not.

\section{CALCULATIONAL METHODS}

\section{A. Computational Details}

The first principles calculations were based on the density functional theory (DFT) as implemented in (Cambridge Serial Total Energy Package) CASTEP code. The plane wave basis sets were expanded in reciprocal space using a kinetic energy cutoff value of $550 \mathrm{eV}$. The Monkhorst-Pack method was employed to generate an $8 \times 8 \times 8$ k-mesh for the energy integrations in the first irreducible part of BZ. For the exchange-correlation energy, the generalized gradient approximation (GGA) of Perdew-Burke-Ernzerhof (PBE) functional was used ${ }^{31}$. The interactions between ionic cores and valence electrons were represented by Norm-conserving pseudo-potentials. For $\mathrm{Y}$, Ta and $\mathrm{O}$ atoms, the valence electron configurations considered are $\mathrm{Y}$ $4 d^{1} 5 s^{2}$, Ta $5 d^{3} 6 s^{2}$ and O $2 s^{2} 2 p^{4}$, respectively. The BFGS (Broyden-Fletcher-Goldfarb-Shannon) optimized method was adopted to obtain the equilibrium crystal structures ${ }^{32}$. The calculations were continued until the total energy changes converged to less than $1.0 \times 10^{-6} \mathrm{eV}$ and the Hellman-Feynman forces acting on distinct atoms converged to less than $0.05 \mathrm{eV} / \AA$.

As part of the calculations we also calculated the ionic and covalent components of the individual bonds based on Mulliken's population analysis ${ }^{33}$. The Mulliken's charge $Q_{m}(A)$ of atom $\mathrm{A}$ and the overlap population $n_{m}(A B)$ for $\mathrm{A}-\mathrm{B}$ bond are defined in the usual way as follows: 


$$
\begin{aligned}
& Q_{m}(A)=\sum_{k} W(k) \sum_{\mu}^{o n A} \sum_{v} P_{\mu \nu}(k) S_{\mu \nu}(k) \\
& n_{m}(A B)=\sum_{k} W(k) \sum_{\mu}^{o n A} \sum_{v}^{o n B} P_{\mu v}(k) S_{\mu v}(k)
\end{aligned}
$$

where $P_{\mu v}(k)$ and $S_{\mu v}(k)$ are the density matrix and the overlap matrix, respectively. $W_{k}$ is the weight associated with the calculated $\mathbf{k}$ points in Brillouin zone. The magnitude and sign of $Q_{m}$ (A) characterizes the iconicity of atom $A$ in the crystal, and $n_{m}(A B)$ can be used to measure the covalent bonding strength approximately.

Since we are interested in the transformation at finite temperatures and constant pressure, the quasi-harmonic approximation (QHA) method ${ }^{34}$ was used throughout to calculate the thermodynamic properties of the compounds at finite temperatures ${ }^{35,36}$. These were computed using the GIBBS code with the thermal properties of solids at constant volume calculated in the QHA from their phonon density of states as a function of frequencies. The phonon contribution to the Helmholtz free energy $F_{\mathrm{ph}}$ is given by

$$
F_{p h}=\frac{1}{2} \sum_{q, v} \hbar \omega_{q, v}+k_{B} T \sum_{q, v} \ln \left[1-\exp \left(-\frac{\hbar \omega_{q, v}}{k_{B} T}\right)\right]
$$

where $\mathbf{q}$ and $\boldsymbol{v}$ are the wave vector and band index, respectively, $\omega_{\mathbf{q}, \boldsymbol{v}}$ is the phonon frequency at $\mathbf{q}$ and $\boldsymbol{v}$, and $T$ is the absolutely temperature. $k_{\mathrm{B}}$ and $\hbar$ are the Boltzmann constant and the reduced Planck constant, respectively. The heat capacity $C_{V}$ and the entropy $S$ at constant volume are given by

$$
C_{v}=\sum_{q, v} k_{B}\left(\frac{\hbar \omega_{q, v}}{k_{B} T}\right)^{2} \frac{\exp \left(\frac{\hbar \omega_{q, v}}{k_{B} T}\right)}{\left[\exp \left(\frac{\hbar \omega_{q, v}}{k_{B} T}\right)-1\right]^{2}}
$$




$$
S=-k_{B} \sum_{q, v} \ln \left[1-\exp \left(-\frac{\hbar \omega_{q, v}}{k_{B} T}\right)\right]-\frac{1}{T} \sum_{q, v} \frac{\hbar \omega_{q, v}}{\exp \left(\frac{\hbar \omega_{q, v}}{k_{B} T}\right)-1}
$$

To compare with experiments, the thermal properties need to be known at constant pressure. These were calculated from the Gibbs free energy $G$ and the $F_{p h}$ using the relationship

$$
G(T, P)={ }_{V}^{\min }\left[U(V)+F_{p h}(T ; V)+P V\right]
$$

where $V$ and $P$ are the volume and pressure, respectively, and $U(V)$ is the total energy, including electronic contributions, at constant volume. The Gibbs free energies at finite temperatures were obtained as the minimum values of the thermodynamic functions, and the corresponding equilibrium volumes and isothermal bulk moduli were obtained simultaneously from the equation of states of the $\mathrm{YTaO}_{4}$ phases. The unit cells used to calculate $U(V)$ and $F_{\mathrm{ph}}(T ; V)$ were relaxed by the first-principles calculations under the hydrostatic-stress conditions. Furthermore, to determine the equilibrium-volume of the unit cells at different temperatures, we employed the Birch-Murnaghan equation of state ${ }^{37}$ to relate the free energy and volume of the tetragonal and monoclinic phases computed at specific volumes and temperatures:

$$
\mathrm{E}(\mathrm{V})=E_{V}+\frac{9 V_{0} B_{0}}{16}\left\{\left[\left(\frac{V_{0}}{V}\right)^{\frac{2}{3}}-1\right]^{3} B_{0}^{\prime}+\left[\left(\frac{V_{0}}{V}\right)^{\frac{2}{3}}-1\right]^{2}\left[6-4\left(\frac{V_{0}}{V}\right)^{\frac{2}{3}}\right]\right\}
$$

where $E(V)$ is the internal energy, $V_{0}$ is the reference volume, $V$ is the deformed volume, $B_{0}$ is the bulk modulus, and $B_{0}{ }^{`}$ is the derivative of the bulk modulus with respect to pressure. In this work, the calculated bulk modulus of monoclinic and tetragonal phase is $183.7 \mathrm{GPa}$ and $128.9 \mathrm{GPa}$, respectively. The $B_{0}{ }^{`}$ is $4.1\left(\mathrm{M}-\mathrm{YTaO}_{4}\right)$ and $4.2\left(\mathrm{~T}-\mathrm{YTaO}_{4}\right)$, and other parameters are tabulated in Table 1, 2 and the parameters for temperature dependent are shown in Fig 3. The results of the 
volume dependent free energies from 200 to $1600{ }^{\circ} \mathrm{C}$ at intervals of $200 \mathrm{C}^{\mathrm{o}}$ are shown in Fig 3 together with the Birch-Murnaghan interpolations.

\section{B. Phonon Calculations}

The phonon spectra of both $\mathrm{YTaO}_{4}$ phases were calculated using the standard (density functional perturbation theory (DFPT) method also implemented in the (Cambridge Serial Total Energy Package) CASTEP code ${ }^{38-40}$. In calculating the phonon dispersion relations and the phonon density of states, a number of approximations were made. First, it was assumed that the mean equilibrium position of each ion is taken to be the positions calculated from the ab-initio calculations at $0 \mathrm{~K}$. Second, it was assumed that the atomic displacements are small compared to the interatomic distances. This leads to a harmonic approximation which is sufficiently accurate to describe most of the lattice dynamical effects of interest. However, further refinement, in the form of anharmonic theory, is required to describe the physical properties at elevated temperatures. The properties of phonons were described using a harmonic approximation based on the knowledge of just the wavevectors and the frequencies $\omega(\mathbf{q})$ and polarization vectors $\mathbf{u}_{\mathbf{k}}$ (q) of phonons with wave vectors $\mathbf{q}$ are determined by the diagonalization of the dynamical matrix $^{41}$ :

$$
\boldsymbol{D}_{k k^{\prime}}^{\alpha \beta}(\mathbf{q})=\frac{1}{\sqrt{m_{k} m_{k}}} \sum_{i} \boldsymbol{\Phi}_{k k^{\prime}}^{\alpha \beta}(0 l) \exp \left[-i \mathbf{q} \cdot\left(\boldsymbol{x}_{0}-\boldsymbol{x}_{l}\right)\right]
$$

which satisfies the eigenvalue equation

$$
\sum_{k^{\prime}} \boldsymbol{D}_{k k^{\prime}}(\mathbf{q}) \boldsymbol{u}_{k^{\prime}}(\mathbf{q})=\omega^{2}(\mathbf{q}) \boldsymbol{u}_{k}(\mathbf{q})
$$


Here an infinite crystal is divided up into primitive cells (labeled by $l$ ) each containing $r$ atoms (labeled by $k$ ) with ionic mass $m_{k}$. The real space interatomic force constants $\boldsymbol{\Phi}_{k k^{\prime}}^{\alpha \beta}\left(\begin{array}{ll}0 & l\end{array}\right)$ include ionic and electronic contributions, so

$$
\boldsymbol{\Phi}_{k k^{\prime}}^{\alpha \beta}\left(\begin{array}{ll}
0 & l
\end{array}\right)=\boldsymbol{\Phi}_{k k^{\prime}}^{\alpha \beta}\left(\begin{array}{ll}
0 & l
\end{array}\right)_{i o n}+\boldsymbol{\Phi}_{k k^{\prime}}^{\alpha \beta}\left(\begin{array}{ll}
0 & l
\end{array}\right)_{e l}
$$

The ionic contribution is calculated from the Ewald sums and the electronic contribution can be expressed as

$$
\boldsymbol{\Phi}_{k k^{\prime}}^{\alpha \beta}(0 \quad l)_{e l}=\int\left[\frac{\partial \rho(\mathbf{r})}{\partial u_{\kappa}^{\alpha}(0)} \frac{\partial V_{i o n}(\mathbf{r})}{\partial u_{\kappa^{\prime}}^{\beta}(l)}+\rho(\mathbf{r}) \frac{\partial^{2} V_{i o n}(\mathbf{r})}{\partial u_{\kappa}^{\alpha}(0) \partial u_{\kappa^{\prime}}^{\beta}(l)}\right] d^{3} r
$$

where $\rho(\mathbf{r})$ is the electron density, $V_{\text {ion }}(r)$ is the ionic potential, and $\partial \rho(\mathbf{r}) / \partial u_{\kappa}^{\alpha}(0)$ represents the density response of the system to a displacement of the $k$ atom in the reference cell $(l=0)$ along the $\alpha$ direction $^{41}$. This linear electron-density response is applied self-consistently in DFPT. The partial (or projected) phonon density of states was determined as a contribution from the given atom to the total phonon DOS. The contribution to the partial density of state on atom $i$, from each phonon band $N_{i}(E)$, was evaluated using:

$$
N_{i}(E)=\int \frac{d \mathbf{k}}{4 \pi^{3}}\left|g_{j}(i)\right|^{2} \Delta\left(E-E_{n}(\mathbf{k})\right)
$$

where $g_{j}$ is the eigenvector (normalized to unit length) associated with the mode of energy $E_{j}$. The projected density of states was then obtained by summation of these individual contributions over all phonon bands.

\section{RESULTS}




\section{A. Free Energies}

The Gibbs free energies calculated at constant pressure for the two $\mathrm{YTaO}_{4}$ phases are shown as a function of temperature in Fig.4 (a). The transition temperature, determined by the intersection point of the two curves, was found to be $1430{ }^{\circ} \mathrm{C}$. This is in excellent agreement with the recent in-situ measurement of the transition temperature by high temperature powder Xray diffraction $\left(1426 \pm 7^{\circ} \mathrm{C}\right)^{42}$. The crystal structures and atomic positions of the two $\mathrm{YTaO}_{4}$ polymorphs were also obtained at different temperatures using the same method. The structural parameters determined at room temperature and $1475{ }^{\circ} \mathrm{C}$ for monoclinic and tetragonal phases are given in Table. 1. The volumes of the unit cells as a function of temperature, Fig 4 (a), indicate a continuous change in volume across the transition. The lattice constants of the two $\mathrm{YTaO}_{4}$ phases were calculated in the QHA using the GIBBS code at different temperatures directly. These are compared in Fig 4 (b) with the values measured by X-ray diffraction at temperature and reported in reference ${ }^{42}$. The agreement between calculated values and experiments is satisfactory as shown in Fig 4 (c). Both the calculated and measured $b_{m}$ exhibit the similar trend as temperature deceases below the transformation temperature. On the other hand, the calculated $a_{m}$ and $c_{m}$ are close to the measured values. Fig 5 shows the calculated cell volume as a function of temperature of the two phases. Note that the volume changes continuously during the phase transition, at least within the resolution of the simulation. The discrepancies between QHA calculations and X-ray diffraction measurements are attributed to a combination of errors in the QHA calculations and presence of lattice defects, such as vacancies and interstitials, which usually produce a larger lattice parameter than calculated.

\section{B. Elastic constants}


The calculated values of the thirteen independent elastic constants are shown in Table 2 and these values were used to calculate the excess Gibbs free energy in Landau theory as described in the following section. Examination of the elastic constants $\left(c_{11}^{o}, c_{22}^{o}\right.$ and $\left.c_{33}^{o}\right)$ indicate that the bonding in the [100] direction is stiffer than that in [010] and [001] directions for the monoclinic phase. Furthermore, the values suggest that the monoclinic phase is weakly anisotropic whereas the tetragonal phase is much more anisotropic. These anisotropies can be expressed in terms of the recently proposed universal anisotropic indices $\left(\mathrm{A}^{\mathrm{U}}\right)^{43}$. The anisotropic index $\left(\mathrm{A}^{\mathrm{U}}\right)$ for monoclinic is 0.445 , typical of many simple oxides whereas that of the tetragonal phase is 5.397 which is unusually high, at least compared with the crystals cataloged by Ranganathan and Ostoja-Starzewski ${ }^{43}$.

\section{Landau free energy parameters}

The macroscopic spontaneous strains were calculated using Eq. (1) based on the experimentally measured lattice parameters. The strains are shown in Fig. 6 indicating that the Landau model satisfactorily describes the ferroelastic transition in the $\mathrm{YTaO}_{4}$ system as the calculated strains closely match the experimental values over a relatively wide range of temperatures, down to one half of the transition temperature (in Kelvin). Above the transition temperature, they all vanish for the tetragonal $\mathrm{YTaO}_{4}$ phase as expected. The Landau excess free energy and $\mathrm{Q}^{2}$ for the monoclinic $\mathrm{YTaO}_{4}$ are also calculated by finding the solutions to the equations 5-8 based on the values of the computed elastic constants and the coupling parameters (shown in Table 3). The results are shown in Fig. 6 (b). Furthermore, the square of the order parameter, $\mathrm{Q}^{2}$, calculated using equation 12 , increases linearly with temperature below $\mathrm{T}_{\mathrm{c}}$ and is above the transition temperature. 


\section{Phonon Spectra}

At the lattice level, a ferroelastic transformation can be driven by the softening of either optical or acoustic modes lying in the Brillouin zone. The calculated phonon dispersion curves and the vibrational density of states of the tetragonal and monoclinic phase are shown in Fig. 7 for their ground states. Neither of the crystal structures in their reference state exhibits a soft mode for any wave vector. They are thus both stable structures, dynamically as well as energetically, in their reference states. On cooling below the transition temperature, however, soft modes appear in the phonon dispersion curves of the tetragonal phase. These are shown in Fig. 8 for three different temperatures below the transition temperature. Comparison of the phonon spectra near the transition temperature show that the tetragonal-to-monoclinic phase transition is caused by the softening of the two transverse acoustic modes in the zone center. These are shown in greater detail in Fig. 9 (a) at the transformation temperature and three lower temperatures for comparison. The vibration pattern is illustrated in Fig. 9 (b) for the first softacoustic modes at the BZ center with symmetry $\mathrm{E}_{\mathrm{u}}$ in the [001] direction. By analyzing the eigenvectors for the soft mode, we find that all the atoms in the crystal structure are simultaneously displaced either in the [001] direction or the [100] direction. We find that for the tetragonal phase, the softening of transverse acoustic mode in the [001] direction leads to an elastic instability associated with shear elastic constants $c_{44}^{0}$ and $c_{66}^{0}$. Meanwhile, the softening of transverse acoustic mode in [100] direction corresponds to the instability of $c_{66}^{0}$. However, our calculations reveal that the elastic instability of tetragonal $\mathrm{YTaO}_{4}$ below the transition temperature is initiated by the softening of $c_{66}^{0}$, which is negative below $\mathrm{T}_{\mathrm{c}}$, since the constant $c_{44}^{0}$ remains positive for all the temperatures. The acoustic phase velocities determined near the Gamma point in the phonon spectra of Figures 7 and $9{ }^{44}$ were used to compute the elastic 
constants with the transverse acoustic (shear waves) corresponding to the constants $c_{44}^{0}, c_{55}^{0}, c_{66}^{0}$ and longitudinal acoustic waves corresponding to the constants $c_{11}^{0}, c_{22}^{0}, c_{33}^{0}$. Above the transition temperature, all the elastic constants of the tetragonal structure are positive.

In general, a good agreement is found between calculated Raman frequency and experimentally measured spectra for the monoclinic phase (Fig 10 and Table 4$)^{45}$. Similar agreement has been reported for the cubic-tetragonal-monoclinic phase transition in $\mathrm{ScVO}_{4}{ }^{15}$. During the tetragonal to monoclinic transition, each $\mathrm{E}_{\mathrm{g}}$ mode splits into two $\mathrm{B}_{\mathrm{g}}$ modes. Moreover, the $\mathrm{A}_{\mathrm{g}}$ and $\mathrm{B}_{\mathrm{g}}$ modes in the high symmetry, tetragonal phase are predicted to convert to other modes with $\mathrm{A}_{\mathrm{g}}$ symmetry in the monoclinic phase. Our observations, up to $1000^{\circ} \mathrm{C}$, show that peaks associated with the two $\mathrm{B}_{\mathrm{g}}$ modes of monoclinic phase approach one another but the thermal background above that temperature prevented us from making unambiguous peak identifications. Therefore, the softening in the $\mathrm{B}_{\mathrm{g}}$ mode could not be conclusively verified.

\section{E. Bond population in phase transition}

The evolution of the relevant Ta-O and Y-O bond's population is collected in Fig 11. Despite the Ta atoms having the same notional four-fold coordination in both the scheelite and fergusonite structures, an analysis of the Ta-O distances across the transition shows that the single Ta-O bond in tetragonal structure splits into two different Ta-O bonds. The changes in lengths of the bonds are tiny but reproducible ${ }^{13}$. Similar results are also identified from the bond population in phase transition by the Mulliken analysis method as shown in Fig 11. It shows the Ta-O bonds are more covalent and $\mathrm{Y}-\mathrm{O}$ bonds more ionic in the crystal structure of $\mathrm{YTaO}_{4}$. 


\section{DISCUSSION}

Our calculations demonstrate that the transition between the high-temperature tetragonal phase of $\mathrm{YTaO}_{4}$ and the lower temperature, monoclinic polymorph is consistent with the transition being a second-order, ferroelastic transition and that the underlying mechanism is associated with phonon softening. The predicted lattice parameters and transformation temperature, as well as the temperature dependence of the symmetry-breaking strains, are all in good agreement with recent measurements providing confidence in the accuracy of the other predictions, such as the identity of the soft modes, the Raman spectra and the elastic constants, that have not yet been determined by experiment. Further confirmation of the transition temperature was obtained by carrying out a series of molecular dynamics calculations of the free energies of the tetragonal and monoclinic phases in the vicinity of the transformation. The free energies, calculated using the Verlet algorithm in the CASTEP suite of programs, are shown in figure $4(\mathrm{~b})$. As expected the free energies are slightly higher on account of the anharmonic contributions to the energies but the transition temperature is unaffected.

Consistent with previous studies of ferroelasticity ${ }^{11,14,23,26}$, we started by assuming that the appropriate form for the Landau free energy was that for a pseudo-proper transformation, as shown in equation 4 . This form allows for the possibility of a structural parameter, such as the tilting of polyhedral structural units, to be represented by order parameter Q as the main driving force for the transformation. However, as seen in table 3, the calculated coupling parameters associated with the symmetry breaking strains, $\lambda_{3}$ and $\lambda_{4}$, are very small. Consequently, the calculated renormalized transition temperature $T_{c}^{*}$ and coefficient $b^{*}$ are essentially the same as $T_{c}$ and $b$, respectively (equation 11 and 12). These results could be mistakenly interpreted as the 
contributions of the symmetry breaking strains to the Landau free energy are negligible. Rather, Q represents the symmetry breaking strains, instead of other structural parameter. The equations compensate the redundant representation of symmetry breaking strains by allowing the associated coupling parameters to be very small. We conclude that the transformation is a proper type, rather than pseudo-proper. This conclusion is also in agreement with the phase transition occurring in both $\mathrm{LaNbO}_{4}$ at $770 \mathrm{~K}^{46}$ and $\mathrm{DyVO}_{4}{ }^{47}$ where the transformation from $4 / \mathrm{m}$ to $2 / \mathrm{m}$ is characterized as proper ferroelastic ${ }^{21}$. This is also consistent with our finding that the temperature dependent order parameter can be represented in terms of the spontaneous breaking strains ${ }^{42}$.

The computed bond lengths also provide some insight into the structural changes observed. In the tetragonal phase, the positions of the cations are fixed by symmetry and the cations planes are spaced exactly by $\mathrm{c} / 4$, along the longest axis Fig.1. As a consequence, the cation polyhedra can only tilt to accommodate the expansion or contraction of the bond lengths during temperature changes. After transitioning to the monoclinic phase, however, both the $\mathrm{Y}$ and Ta polyhedra have an additional degree of freedom, and can translate along the $b$-axis of the monoclinic (corresponding to the tetragonal $c$-axis). This translational freedom allows the $\mathrm{Y}$ and Ta planes to move relative to one another as shown in the crystal structure of Fig. 12. At the same time, as the temperature decreases, the oxygen ions in the monoclinic phase become increasingly aligned in their closed packed planes. Adjacent, closed-packed planes shear relative to one another, increasing the monoclinic angle with decreasing temperature below the transition temperature (Fig 12). This suggests that the monoclinic angle can be used as an experimentally determinable order parameter. Indeed, our data for the monoclinic angle, $\Delta \beta=\beta-90$, shows a 
power law exponent of $1 / 2$ in accord with the Landau free energy expansion down to about $700{ }^{\circ} \mathrm{C}$ 42.

The Mulliken population analyses clearly indicate the contrasting covalent nature of the Ta-O and the $\mathrm{Y}-\mathrm{O}$ ionic bonding, which is not surprising. More revealing, however, is that the effective coordination number $(\mathrm{ECN})$ of the cations changes during the transformation. Using a weighted sum scheme ${ }^{48}$ in which the anions are counted as fractional numbers, from 1 for the nearest anion, decreasing to zero as the anion distance increases, the effective coordination number of the tantalum ions are 4.0 and 4.3, in the tetragonal and monoclinic phases, respectively. The effective coordination of 4.0 for the tantalum ions in the tetragonal phase corresponds to the notional coordination of $\mathrm{Ta}$ in $\mathrm{a} \mathrm{TaO}_{4}$ tetrahedron but the larger number in the monoclinic phase indicates that the $\mathrm{TaO}_{4}$ tetrahedra are distorted. This is consistent with the calculations indicating that one pair of Ta-O bonds became elongated while the other pair contracts. Fig.1 The latter, in turn, allows other nearby oxygen atoms to move closer to the central Ta ion contributing to the slight increase in the coordination number. Essentially, the $\mathrm{TaO}_{4}$ tetrahedra become less symmetric and more densely packed in the monoclinic phase than in the tetragonal phase. The effective coordination number of the yttrium ions only decreases slightly from 8.0 in the tetragonal phase to 7.92 in the monoclinic phases, which is probably not significant.

One of our findings is that the transformation is associated with a soft acoustic mode at the BZ center with a symmetry of $\mathrm{E}_{\mathrm{u}}$ in the [001] direction. This contrasts with the $\mathrm{B}_{\mathrm{g}}$ soft mode that has been reported for similar compounds, $\mathrm{ScVO}_{4}{ }^{15}, \mathrm{YVO}_{4}{ }^{49}, \mathrm{BiVO}_{4}{ }^{50,51}$ and $\mathrm{YNbO}_{4}$, which have the same space groups and point group, and which also undergo a tetragonal (scheelite) and monoclinic (fergusonite) transformation. As the radius, mass and positions of the individual 
atoms, as well as their local environment and symmetry, can all affect the vibrational energies, we also calculated the vibrational modes of $\mathrm{YVO}_{4}$ and $\mathrm{YNbO}_{4}$, again from first principles, and found that they have the $\mathrm{B}_{\mathrm{g}}$ soft mode, consistent with previous reports. However, for the $\mathrm{Yb}$ analog, $\mathrm{YbTaO}_{4}$, the transformation is associated with the $\mathrm{A}_{\mathrm{u}}$ symmetry of an acoustic mode at the center of the Brillouin zone. A comparison of the energy ranges of the vibrational modes for the four compounds with the Fergusonite structure is listed in Table 5. As can be seen from the energies of the allowable vibrational modes, summarized in the table, while the high energy modes of the four compounds have the same character, this is not the case when comparing the low energy modes. For these, there is greater similarity between the compounds with the heavier, Ta ion in the B-site, than with $\mathrm{YNbO}_{4}$. These differences cannot be explained in terms of ionic radii since the radius of $\mathrm{Nb}^{5+}$ and $\mathrm{Ta}^{5+}$ are almost identical in the same coordination but we note that tantalum has a higher atomic weight. Further work is clearly needed to establish the reason for these differences in soft-mode behavior.

According to group theory the ferroelastic phase transition results in the formation of two equivalent variants of equal free energy. This can be seen from equation 7 and 9 , where the sign of symmetry-breaking strains $e_{1}-e_{2}$ and $e_{6}$ change sign when the order parameter changes sign. The schematic diagram of the transitions along with the order parameter is shown in Fig. 12. In the parent tetragonal phase, the basal plane separation are equal in length (i.e., $a$ and $b$ ) and the closed-packed oxygen planes are also equal in both the $a$ or $b$ directions. The first variant orientation occurs in response to the strains $e_{1}$ and $-e_{2}$ acting along the $a$ and $b$-axis of tetragonal, respectively, and the shear strain acting around the $-c$ axis. After the transformation, the tetragonal $b$-axis becomes the $c$-axis in the monoclinic and the resulting monoclinic structure has closed packed oxygen plane perpendicular to the tetragonal $b$-axis. The orientation of the second 
variant emerges when the strains $e_{,}-e_{2}$, and $e_{6}$ operate in the opposite direction, i.e., along $b$ and $a$ axes, and around $c$ axis of tetragonal, respectively, as illustrated in Fig 12. Thus the tetragonal $a$-axis becomes the $c$-axis in the monoclinic and the closed packed oxygen plane in the monoclinic structure is perpendicular to the tetragonal $a$-axis. In the absence of any structural heterogeneity, both directions are equally favorable and consequently both monoclinic variants should exist at the same volume fraction. Since $a$ and $b$ are at the right angles, these monoclinic variants are consequently should be related by $90^{\circ}$ rotation plus an offset determined by strain energy associated by the monoclinic deformation. This rotation can be clearly seen from the difference in the orientation of closed-packed oxygen plane in each monoclinic variant. Additionally, opposite sides of the basal plane, i.e., $c$ and $a$, should meet and therefore are strained at the boundary between the variants or domains. This analysis is supported by recent TEM observations of twin boundaries with rotational relation of $95.05^{\circ}$ between the domains ${ }^{42}$.

\section{CONCLUSIONS}

First-principles calculations show that the tetragonal-to-monoclinic phase transition in $\mathrm{YTaO}_{4}$ system is a second order, ferroelastic phase transition. The calculated transition temperature using first principles agrees well with the observed transition temperature recently reported by high temperature X-ray diffraction. In addition, the Landau excess free energy for the monoclinic phase was derived from a "broken symmetry" model of tetragonal $\mathrm{YTaO}_{4}$. The calculated spontaneous macroscopic strains using Landau's method also agree well with the experimental results. From the calculated phonon spectra, it can be concluded that the tetragonalto-monoclinic phase transition is driven by the softening of transverse acoustic modes having $\mathrm{E}_{\mathrm{u}}$ symmetry in the $\mathrm{BZ}$ center rather than the Raman-active $\mathrm{B}_{\mathrm{g}}$ mode at the same $\mathbf{k}$ point. The $\mathrm{TaO}_{4}$ tetrahedra tilt produces not only a shearing to the monoclinic structure but also alters the 
separation of the cation planes in the c direction. Using a Mulliken analysis, the Ta-O bonds are predominately covalent and the Y-O bonds ionic. The Raman modes in the tetragonal and monoclinic $\mathrm{YTaO}_{4}$ have been calculated and, in general, there is a good agreement with experimental data for the monoclinic phase.

\section{ACKNOWLEDGEMENTS:}

This work was supported at Harvard University by a grant from ONR number N00014-012-10993. We are indebted to Mary Gurak for making the Raman measurements. 


\section{References:}

1 E. Gregoryanz, R. J. Hemley, H. K. Mao, and P. Gillet, Phys Rev Lett 84, 3117-3120 (2000).

2 E. Salje, Annual Review of Materials Research 42, 265-283 (2012).

3 M. T. Dove, Phase Transitions 61, 1-17 (1997).

4 E. K. H. Salje, S. A. Hayward and W. T. Lee, Acta Crystallographica Section A 61, 3-18 (2005).

$5 \quad$ K. Aizu, Phys Rev B 2, 754-772 (1970).

6 A. V. Virkar and R. Matsumoto, J Am Ceram Soc 69, C224-C226 (1986).

7 C. Mercer, J. R. Williams, D. R. Clarke, and A. G. Evans, Proc R Soc A 463, 1393-1408 (2007).

8 J. Chevalier, L. Gremillard, A. V. Virkar, and D. R. Clarke, J Am Ceram Soc 92, 19011920 (2009).

9 C. Chan, F. F. Lange, M. Rühle, J. Jue, and A. V. Virkar, J Am Ceram Soc 74, 807-813 (1991).

10 D. Baither, M. Bartsch, B. Baufeld, A. Tikhonovsky, A. Foitzik, M. Rühle, and U. Messerschmidt, J Am Ceram Soc 84, 1755-1762 (2001).

11 M. A. Carpenter, R. J. Hemley and H. K. Mao, J Geophys Res 105, 10807 - 10816 (2000).

12 W. W. Cao, A. Saxena and D. M. Hatch, Phys Rev B 64, 0241062 (2001).

13 N. Choudhury and S. L. Chaplot, Phys Rev B 73, 0943049 (2006).

14 A. Togo, F. Oba and I. Tanaka, Phys Rev B 78, 13410613 (2008).

15 V. Panchal, F. J. Manjon, D. Errandonea, P. Rodriguez-Hernandez, J. Lopez-Solano, A. Munoz, S. N. Achary, and A. K. Tyagi, Phys Rev B 83 (2011).

16 M. T. Dove and S. Redfern, Am Min 82, 8-15 (1997).

17 M. Basta and A. Sieradzki, Phase Transitions 83, 235-243 (2010).

18 J. A. Valgoma, J. M. Perez-Mato, A. Garcia, K. Schwarz, and P. Blaha, Phys Rev B 65 (2002).

19 S. Ono and K. Mibe, Phys Rev B 84, 0541145 (2011). 

(2013).

E. K. H. Salje, Phase Transitions in Ferroelastic and Co-elastic Crystals (Cambridge University Press, Cambridge, 1990).

22 M. M. Fejer, B. A. Auld and P. Toledano, Phys Rev B 27, 5717-5746 (1983).

23 M. A. Carpenter and E. Salje, Eur J Mineral 10, 693-812 (1998).

M. A. Carpenter, E. Salje and A. Graeme-Barber, Eur J Mineral 10, 621-691 (1998).

G. Errandonea, Phys Rev B 21, 5221-5236 (1980).

D. Errandonea, R. Kumar, J. Lopez-Solano, P. Rodriguez-Hernandez, A. Munoz, M. G. Rabie, and R. S. Puche, Phys Rev B 83 (2011).

V. S. Stubi V C An, J Am Ceram Soc 47, 55--58 (1964).

L. Jian and C. M. Wayman, J Am Ceram Soc 80, 803-806 (1997).

L. Jian and C. M. Wayman, J Am Ceram Soc 79, 1642-1648 (1996).

L. Truskinovsky, G. Zanzotto and G. Fadda, Phys Rev B 66, 174107 (2002).

J. P. Perdew, K. Burke and M. Ernzerhof, Phys Rev Lett 77, 3865-3868 (1996).

J. D. Head and M. C. Zerner, Chem Phys Lett 122, 264-270 (1985). (1996).

S. Baroni, P. Giannozzi and E. Isaev; Vol. 71 (Mineralogical Soc Amer, Chantill, 2010), p. 39-57.

35 A. Seko, F. Oba, A. Kuwabara, and I. Tanaka, Phys Rev B 72 (2005).

36 M. A. Blanco, E. Francisco and V. Luana, Computer Phys Comm 158, $57-72$ (2004).

37 F. Birch, Phys Rev 71, 809-824 (1947).

38 M. Segall, P. Lindan, M. Probert, C. Pickard, P. Hasnip, S. Clark, and M. Payne, J Phys Condens Matter 14, 2717 (2002).

39 P. Hohenberg and W. Kohn, Phys Rev 136, B864 (1964).

40 S. Baroni, S. de Gironcoli, A. Dal Corso, and P. Giannozzi, Rev Mod Phys 73, 515-562 (2001).

41 R. M. Wentzcovitch, S. de Gironcoli, S. Baroni, and B. B. Karki, Phys Rev B 61, 8793$8800(2000)$.

42 S. Shian, P. Sarin, M. Gurak, M. Baram, W. M. Kriven, and D. R. Clarke, Acta 
Materialia 69, 196-202 (2014).

43 S. I. Ranganathan and M. Ostoja-Starzewski, Phys Rev Lett 101, 055504 (2008).

44 P. Halevi, G. Martínez, L. Dobrzynski, B. Djafari-Rouhani, and M. S. Kushwaha, Phys Rev B 49, 2313-2322 (1994).

45 G. Blasse, J Solid State Chem 7, 169-171 (1973).

46 L. H. Brixner, J. F. Whitney, F. C. Zumsteg, and G. A. Jones, Materials Research Bulletin 12, 17-24 (1977).

47 W. Rehwald, Advances in Physics 22, $721-755$ (1973).

48 C. Ferraris, Fundamentals of Crystallography, 3nd ed. (Oxford University Press, Oxford, 2011).

49 F. J. Manjon, P. Rodriguez-Hernandez, A. Munoz, A. H. Romero, D. Errandonea, and K. Syassen, Phys Rev B 81, 075202 (2010).

50 E. S. Fisher, J Phys Cond Matter 1, 2875-2890 (1989).

51 A. Pinczuk, G. Burns and F. H. Dacol, Solid State Comm 24, 163-165 (1977). 


\section{Figures Captions}

Figure 1. Projections of (a) the tetragonal structure at $1475^{\circ} \mathrm{C}$ (space group $\mathrm{I} 4_{1} / \mathrm{a}$, No.88, point group $\mathrm{C}_{4 h}^{6}$ ) and (b) the monoclinic structure at $20^{\circ} \mathrm{C}$ (space group $\mathrm{I}_{12} / \mathrm{a} 1$, No. 15 , point group $\mathrm{C}^{6}{ }_{2 \mathrm{~h}}$ ) of $\mathrm{YTaO}_{4}$. Comparison of the positions of the ions in the a-projection (top row) indicates that in in the tetragonal phase both the Ta and $\mathrm{Y}$ atoms lie in the same a-b plane and the planes are equidistant along the c-axis. In the monoclinic phase, however, each $\mathrm{Ta}$ and $\mathrm{Y}$ atoms are slightly in different planes and each plane is not equidistant along the longest axis (b-axis of monoclinic). In addition, each $\mathrm{Ta}-\mathrm{O}$ bond in the $\mathrm{TaO}_{4}$ tetrahedra has equal length in the tetragonal structure whereas they have slightly different lengths in the monoclinic structure (bottom row).

Figure 2. The relations between the symmetry-breaking strains $\left(e_{1}-e_{2}\right.$ and $\left.e_{6}\right)$ and the transformation of the tetragonal to monoclinic unit cells in the Cartesian coordinate system. Note the differences in the axes before and after the transformation.

Figure 3. Volume dependence of the free energy of tetragonal and monoclinic phases of $\mathrm{YTaO}_{4}$ from 200 to $1600{ }^{\circ} \mathrm{C}$ at $200{ }^{\circ} \mathrm{C}$ intervals calculated using the GIBBS code ${ }^{36}$. The solid symbols are calculated values and the solid lines are the fittings using the Birch-Murnaghan equation of states.

Figure 4 (a): The computed Gibbs free energies of the tetragonal and monoclinic $\mathrm{YTaO}_{4}$ phases as a function of temperature. The intersection of the two curves indicates that the transition 
temperature is close to $1430{ }^{\circ} \mathrm{C}$. As described in the text, the Gibbs free energy of T-/M- $\mathrm{YTaO}_{4}$ compound was evaluated using Debye's quasi-harmonic approximation (QHA), as implemented in GIBBS $\operatorname{code}^{36}$. (b): Comparison of the free energies of the two phases calculated using the QHA and ab-initio MD methods in the vicinity of the transition temperature. The differences in energy are small indicating that the anharmonic contribution to the total free energy of the two phases is also small. There is no discernable effect on the calculated transition temperature. (c) The calculated (hollow symbols) and measured (solid symbols) lattice parameters of the monoclinic and tetragonal $\mathrm{YTaO}_{4}$ phases as a function of temperature. Note that, the calculated transition temperature $\left(1430^{\circ} \mathrm{C}\right)$ agrees well with the experimental value $\left(1426 \pm 7^{\circ} \mathrm{C}\right) .{ }^{42}$

Figure 5. The calculated cell volume as a function of temperature of the $\mathrm{M}-\mathrm{YTaO}_{4}$ (blue squares represent low-temperature monoclinic phase) and $\mathrm{T}-\mathrm{YTaO}_{4}$ (red circles represent hightemperature tetragonal phase) phases. The dash and the solid lines represent the imaginary and the actual volumes, respectively. Color online.

Figure 6 (a) The spontaneous macroscopic strains indicated by the symbols calculated using Eq. (2) with the experimental lattice constants from reference ${ }^{42}$. The solid lines represent the strains calculated from lattice constants from first principles calculations. (b) The Landau excess free energy and the corresponding square of order parameters $Q$ are shown as a function of temperature. The dashed vertical line refers to the transition temperature. 
Figure 7. The phonon dispersion curves and the density of states of the ground states of the monoclinic and tetragonal phases.

Figure 8 Phonon dispersion curves of tetragonal $\mathrm{YTaO}_{4}$ at progressively low temperature, below the transition temperature: (a) $1400^{\circ} \mathrm{C}$, (b) $1250^{\circ} \mathrm{C}$, and (c) $850^{\circ} \mathrm{C}$. The soft modes are indicated by the negative values.

Figure 9 (a) The softening of one of the transverse-acoustic modes in the [001] direction of tetragonal phase near the center of BZ at different temperatures. (b) The vibrational directions corresponding to the soft-transverse acoustic mode at the zone center with irreducible representation $\mathrm{E}_{\mathrm{u}}$. Note: all arrows point toward $\left(\begin{array}{lll}0 & 0 & -1\end{array}\right)$ and their size indicate relative amplitudes of the vibration. The color and sizes of the atoms are the same as shown in Fig 1.

Figure 10. Room temperature Raman spectrum of monoclinic $\mathrm{YTaO}_{4}$. The peak locations are determined by peak fitting (bold) and are compared with calculation (in parentheses and Red) as shown in Table 4. In general, the calculated peaks locations are in good agreement with the experimental data, with the exception of the peaks located at 671 and $705 \mathrm{~cm}^{-1}$. The laser excitation was $532 \mathrm{~nm}$ and the lowest energy peak at $119 \mathrm{~cm}^{-1}$ may had been partially truncated by the laser filter.

Figure 11. Population of the cation-oxygen bonds as a function of temperature for the tetragonal and monoclinic phases. The Ta-O and $\mathrm{Y}-\mathrm{O}$ bonds have covalent and ionic characteristics, respectively, at all temperatures. 
Figure 12. The Landau free energy, $G$ ', as a function of order parameter, $Q$, plotted at different temperatures. The relation of the two monoclinic variant relative to the parent tetragonal phases and associated symmetry breaking strains are also shown. Note the location of the unit cell and the orientation of closed-packed plane of oxygen (shown as dashed lines) in each monoclinic variants with respect to the parent tetragonal axis. 
Figures and Tables

Figures

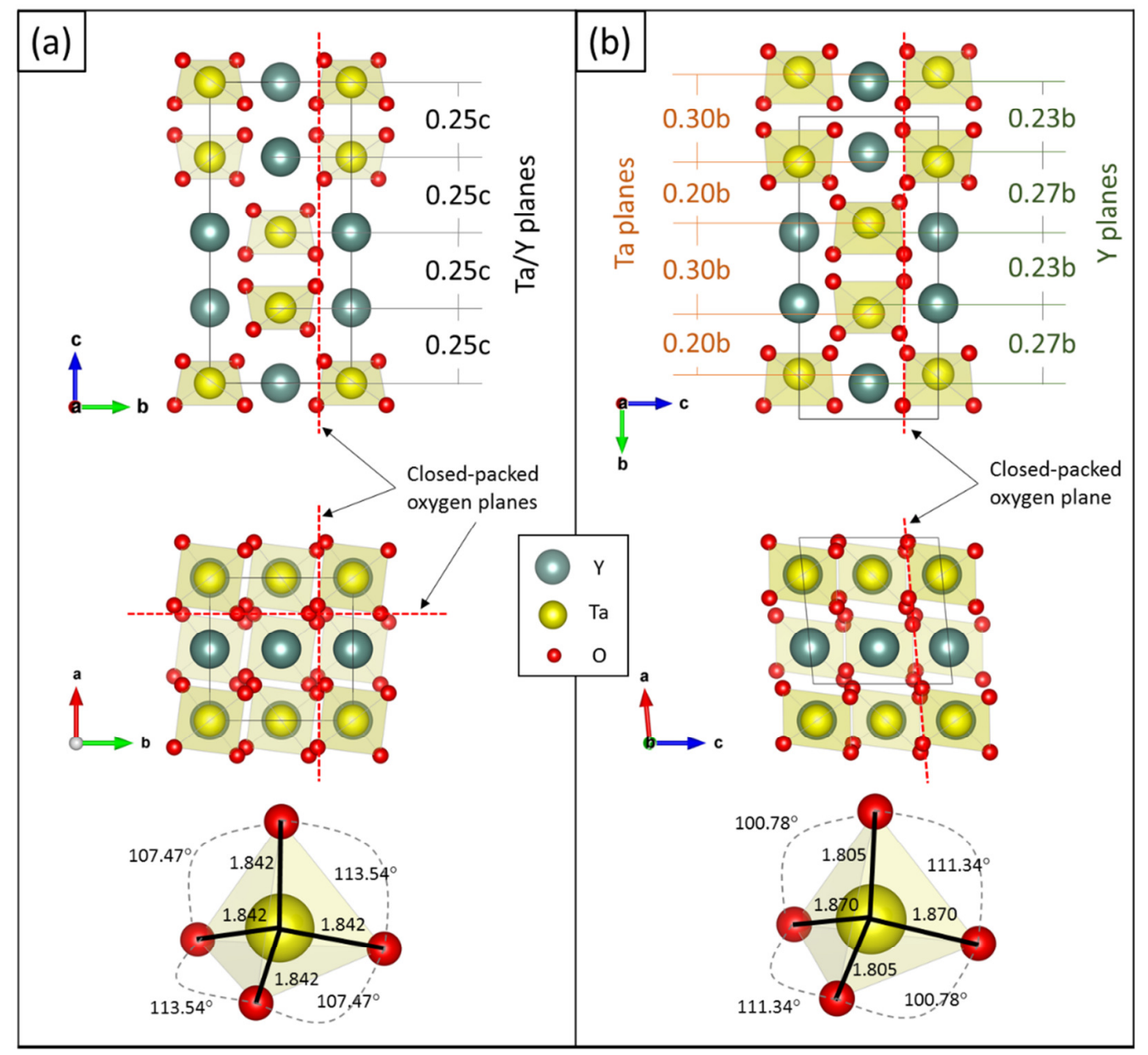

Fig 1. Projections of (a) the tetragonal structure at $1475^{\circ} \mathrm{C}$ (space group $\mathrm{I} 4_{1} / \mathrm{a}$, No.88, point group $\mathrm{C}_{4 \mathrm{~h}}^{6}$ ) and (b) the monoclinic structure at $20^{\circ} \mathrm{C}$ (space group $\mathrm{I}_{12} / \mathrm{a} 1$, No. 15 , point group $\mathrm{C}^{6}{ }_{2 \mathrm{~h}}$ ) of $\mathrm{YTaO}_{4}$. Comparison of the positions of the ions in the a-projection (top row) indicates that in in the tetragonal phase both the Ta and $\mathrm{Y}$ atoms lie in the same a-b plane and the planes are equidistant along the c-axis. In the monoclinic phase, however, each Ta and $\mathrm{Y}$ atoms are slightly in different planes and each plane is not equidistant along the longest axis (b-axis of monoclinic). In addition, each $\mathrm{Ta}-\mathrm{O}$ bond in the $\mathrm{TaO}_{4}$ 
tetrahedra has equal length in the tetragonal structure whereas they have slightly different lengths in the monoclinic structure (bottom row). 


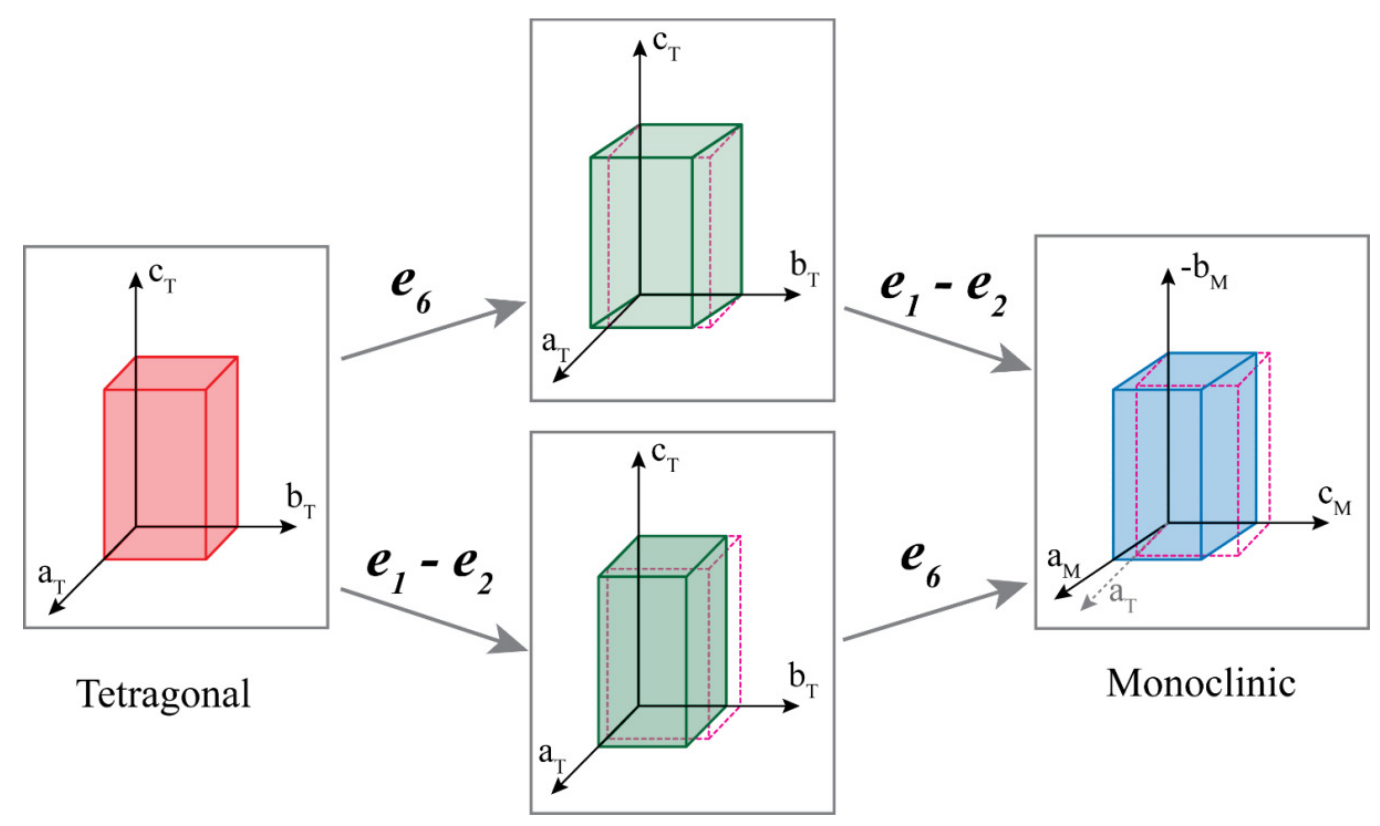

Fig.2. The relations between symmetry-breaking strains $\left(e_{1}-e_{2}\right.$ and $\left.e_{6}\right)$ and the transformation of tetragonal to monoclinic unit cells in the Cartesian coordinate system. Note the differences in the axes before and after the transformation. 


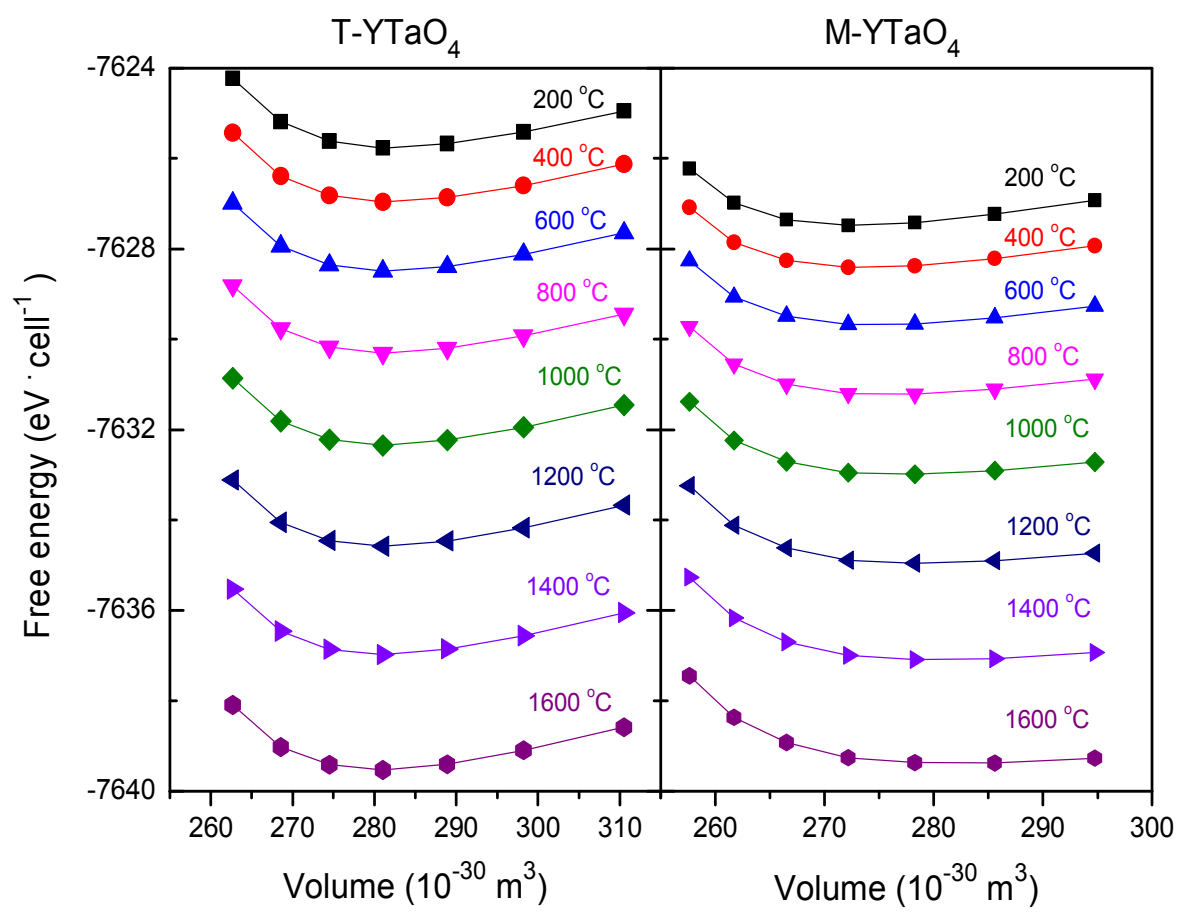

Fig 3. Volume dependence of the free energy of tetragonal and monoclinic phases of $\mathrm{YTaO}_{4}$ from 200 to $1600{ }^{\circ} \mathrm{C}$ at $200{ }^{\circ} \mathrm{C}$ intervals calculated using the GIBBS code ${ }^{36}$. The solid symbols are calculated values and the solid lines are the fittings using the Birch-Murnaghan equation of states. 

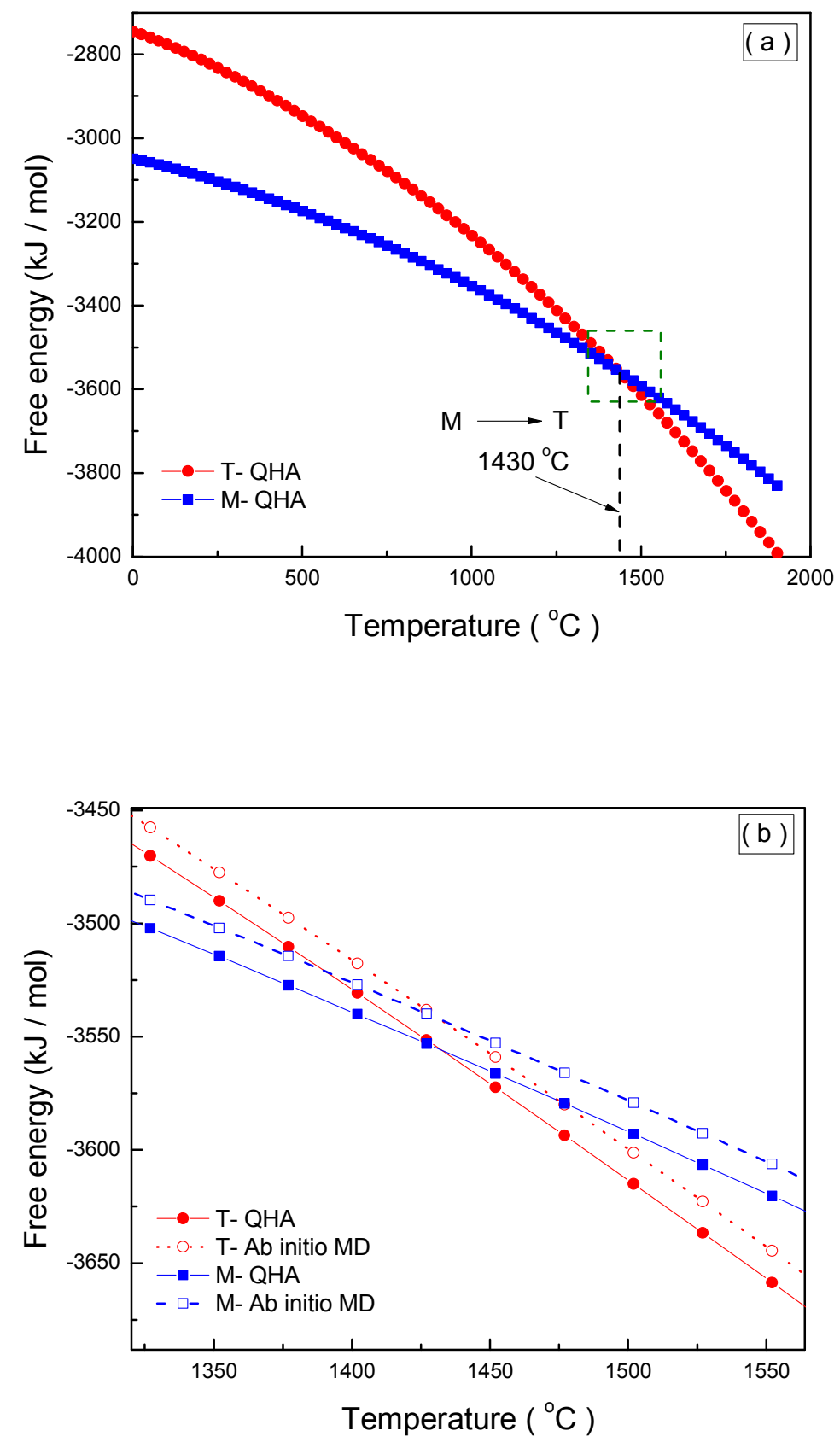


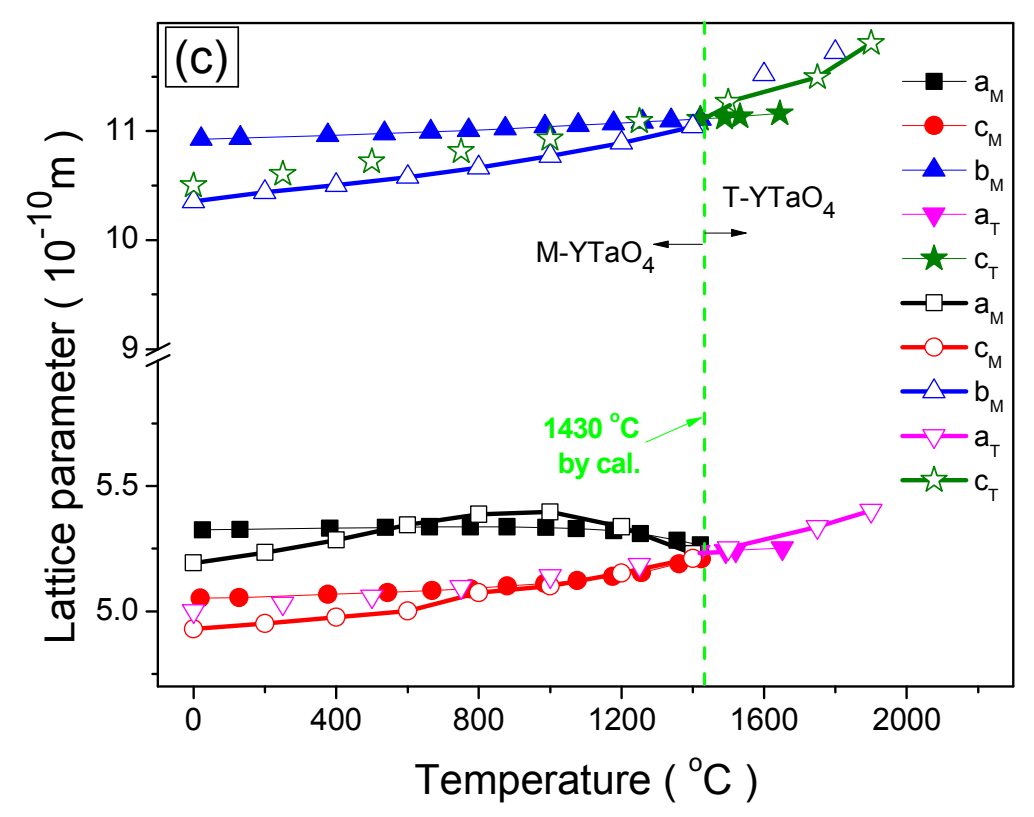

Fig 4 (a): The computed Gibbs free energies of the tetragonal and monoclinic $\mathrm{YTaO}_{4}$ phases as a function of temperature. The intersection of the two curves indicates that the transition temperature is close to $1430{ }^{\circ} \mathrm{C}$. As described in the text, the Gibbs free energy of T-/M- $\mathrm{YTaO}_{4}$ compound was evaluated using Debye's quasi-harmonic approximation (QHA), as implemented in GIBBS code ${ }^{36}$. (b): Comparison of the free energies of the two phases calculated using the QHA and ab-initio MD methods in the vicinity of the transition temperature. The differences in energy are small indicating that the anharmonic contribution to the total free energy of the two phases is also small. There is no discernable effect on the calculated transition temperature. (c): The calculated (hollow symbols) and measured (solid symbols) lattice parameters of the monoclinic and tetragonal $\mathrm{YTaO}_{4}$ phases as a function of temperature. Note that, the calculated transition temperature $\left(1430^{\circ} \mathrm{C}\right)$ agrees well with the experimental value $\left(1426 \pm 7^{\circ} \mathrm{C}\right) .{ }^{42}$ 


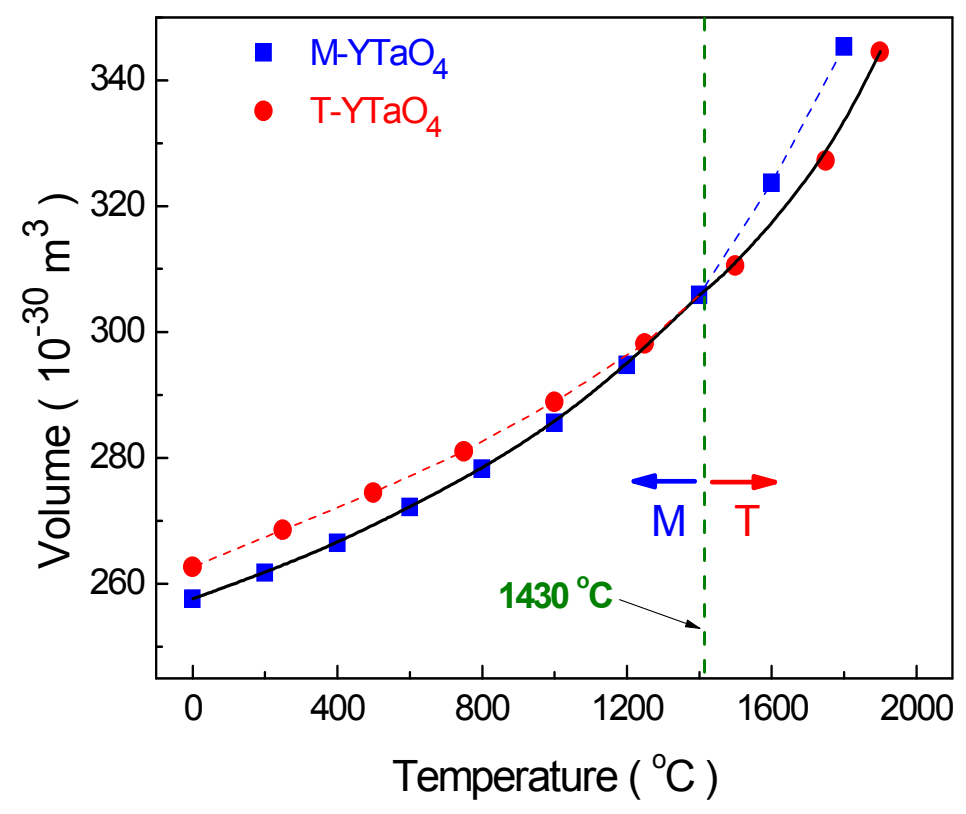

Fig 5. The calculated cell volume as a function of temperature of the $\mathrm{M}-\mathrm{YTaO}_{4}$ (blue squares represent low-temperature monoclinic phase) and $\mathrm{T}-\mathrm{YTaO}_{4}$ (red circles represent high-temperature tetragonal phase) phases. The dash and the solid lines represent the imaginary and the actual volumes, respectively. Color online. 

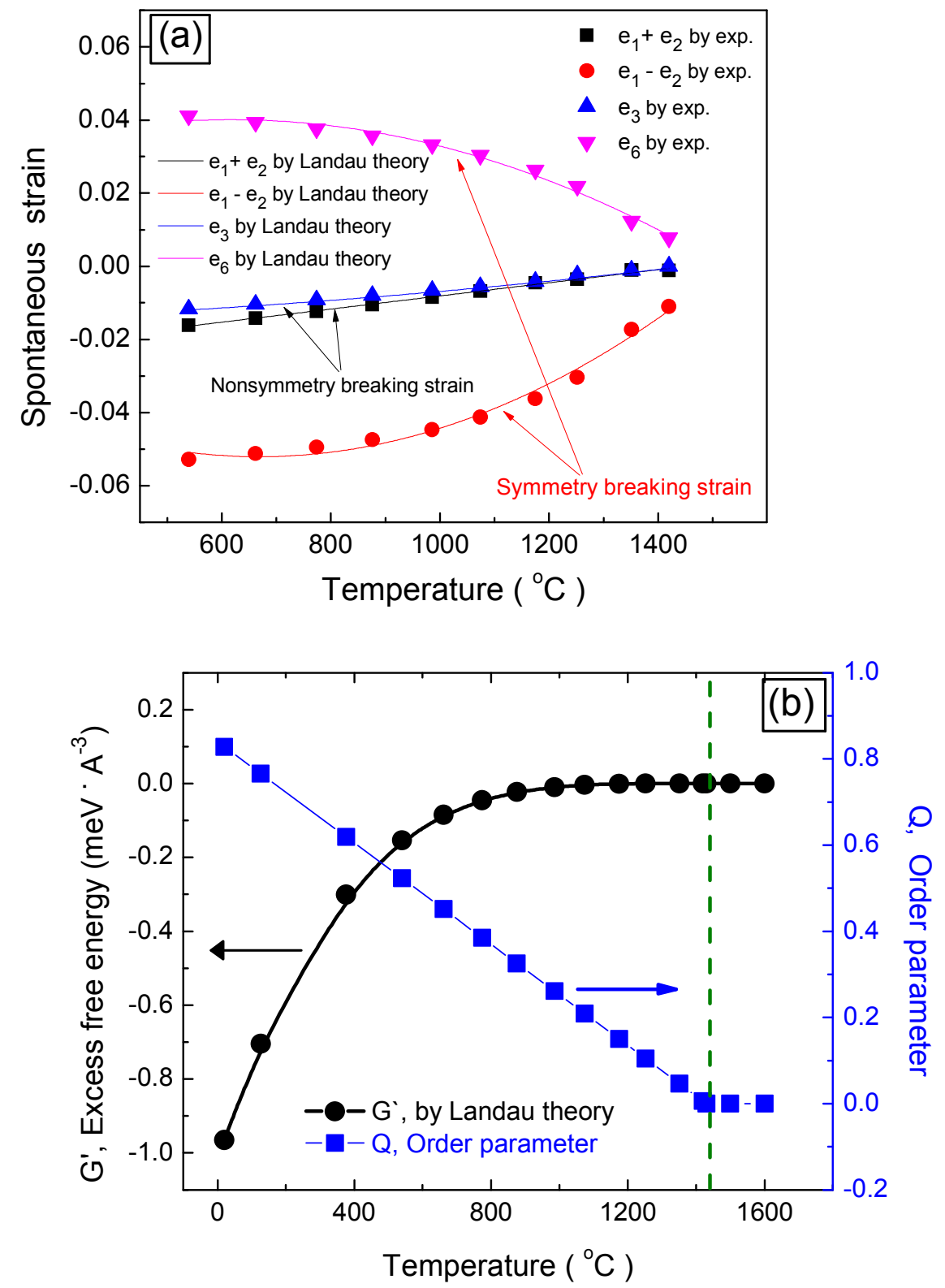

Fig. 6 (a) The spontaneous macroscopic strains indicated by the symbols calculated using Eq. (2) with the experimental lattice constants from reference ${ }^{42}$. The solid lines represent the strains calculated from lattice constants from first principles calculations. (b) The Landau excess free energy and the corresponding square of order parameters $Q$ are shown as a function of temperature. The dashed vertical line refers to the transition temperature. 

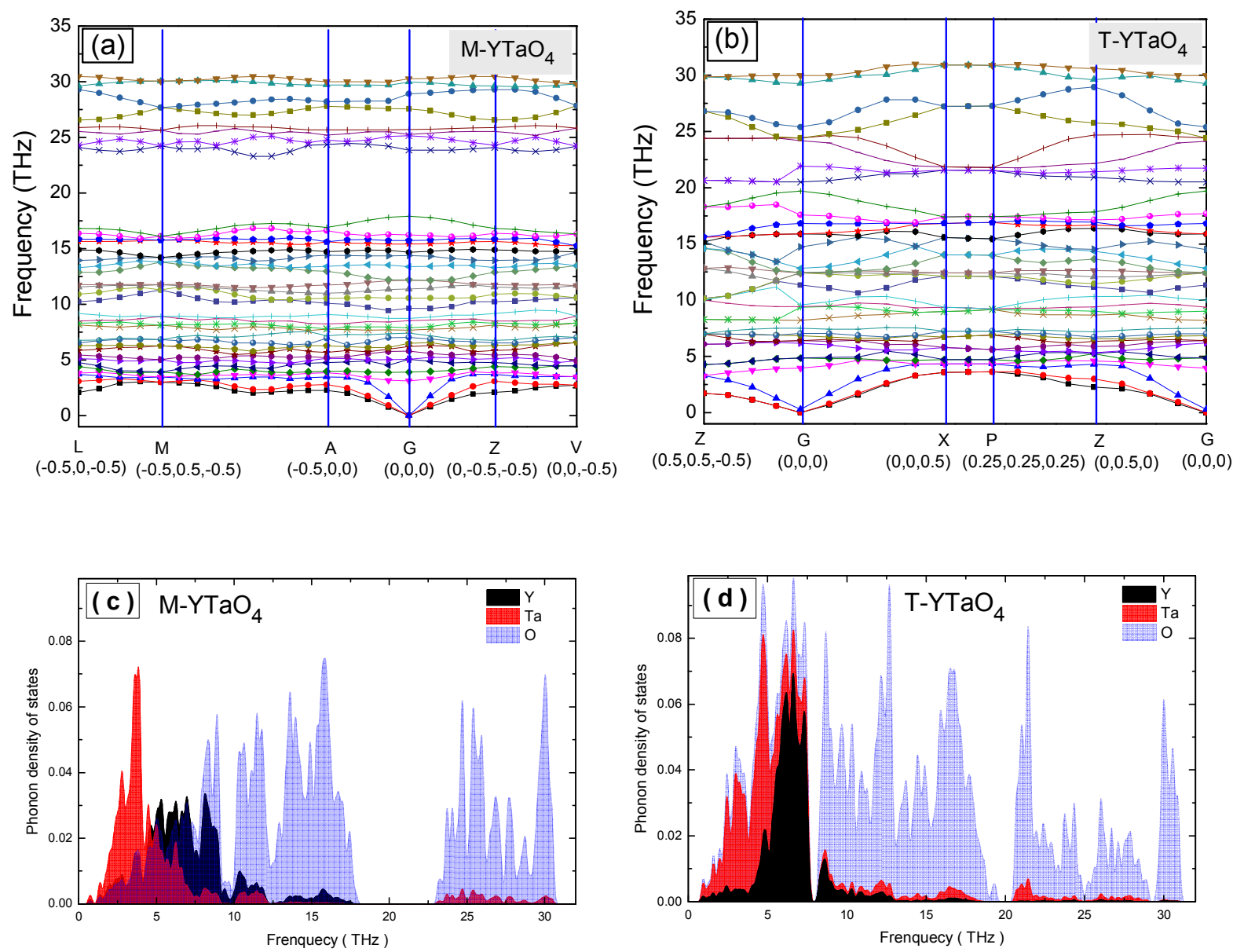

Fig 7. The phonon dispersion curves and the density of states of the ground states of the monoclinic and tetragonal phases. 

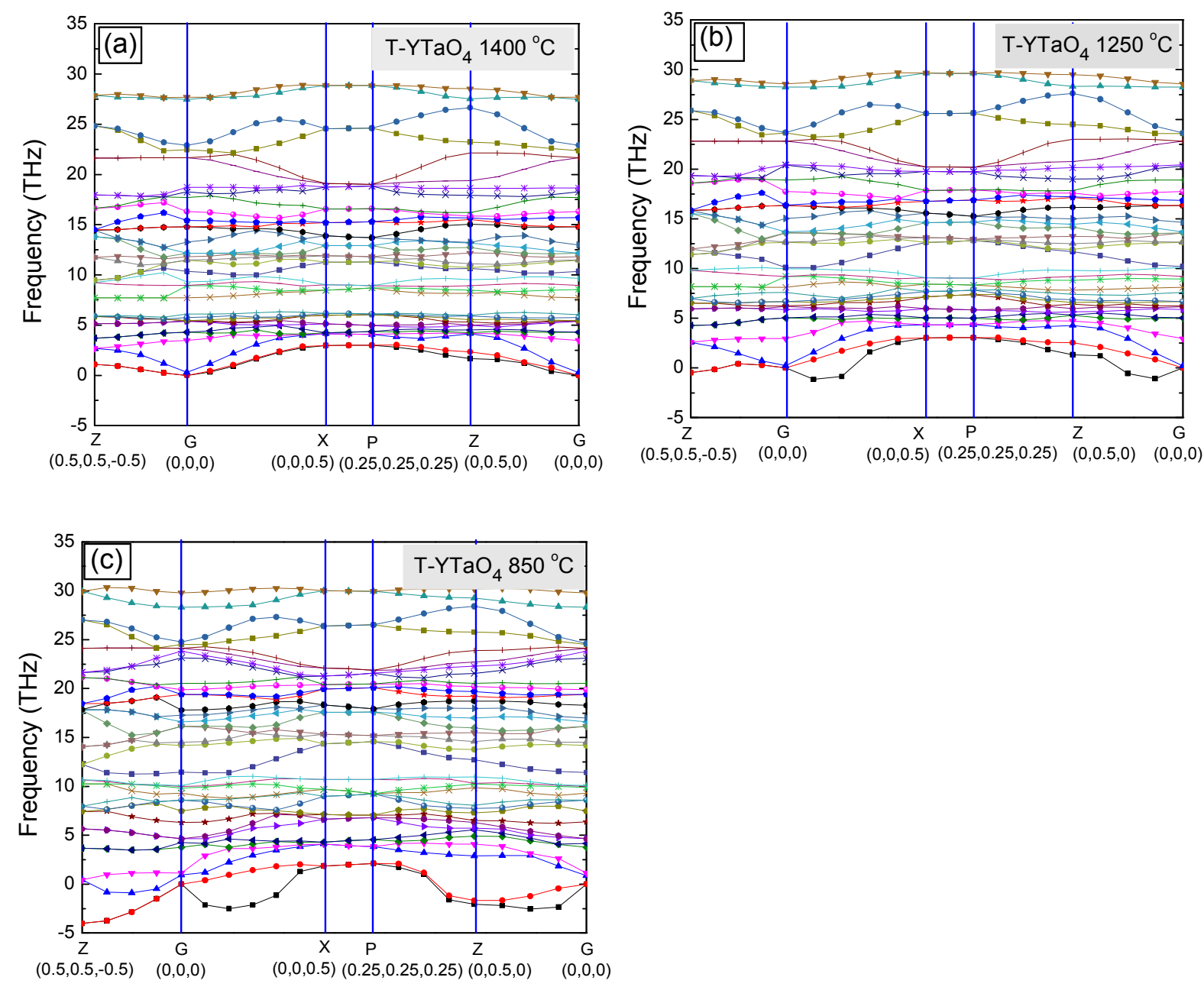

Fig 8 Phonon dispersion curves of tetragonal $\mathrm{YTaO}_{4}$ at progressively low temperature, below the transition temperature: (a) $1400^{\circ} \mathrm{C}$, (b) $1250^{\circ} \mathrm{C}$, and (c) $850^{\circ} \mathrm{C}$. The soft modes are indicated by the negative values. 

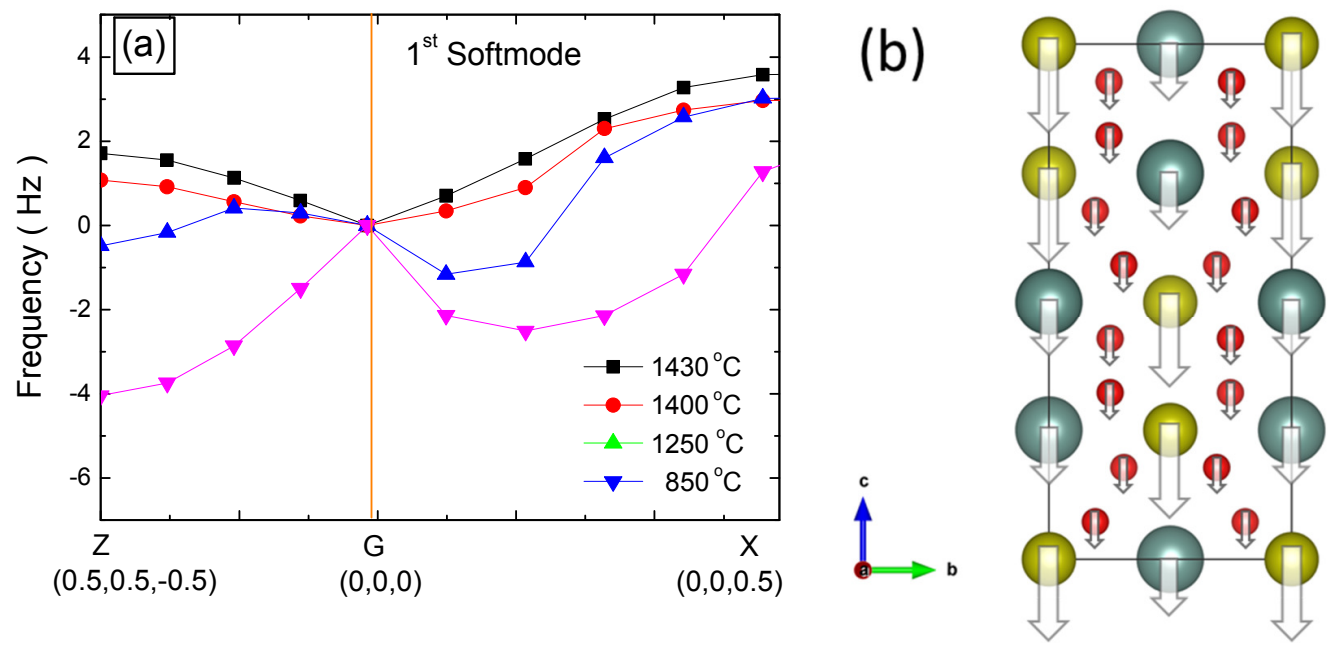

Fig. 9 (a) The softening of one of the transverse-acoustic modes in the [001] direction of tetragonal phase near the center of $\mathrm{BZ}$ at different temperatures. (b) The vibrational directions corresponding to the softtransverse acoustic mode at the zone center with irreducible representation $\mathrm{E}_{\mathrm{u}}$. Note all arrows point toward $\left(\begin{array}{lll}0 & 0 & -1\end{array}\right)$ and their size indicate relative amplitudes of the vibration. The color/size of atoms are defined in Fig 1. 


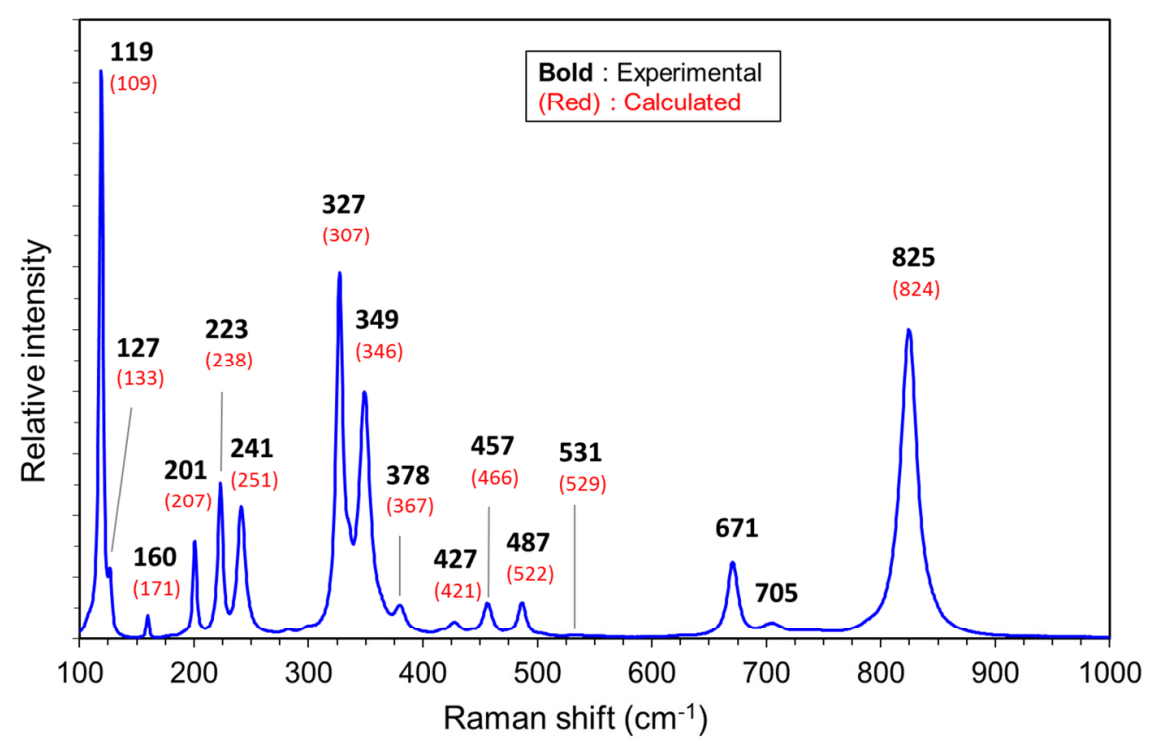

Fig 10. Room temperature Raman spectrum of monoclinic $\mathrm{YTaO}_{4}$. The peak locations are determined by peak fitting (bold) and are compared with calculation (in parentheses and Red) as shown in Table 4. In general, the calculated peaks locations are in good agreement with the experimental data, with the exception of the peaks located at 671 and $705 \mathrm{~cm}^{-1}$. The laser excitation was $532 \mathrm{~nm}$ and the lowest energy peak at $119 \mathrm{~cm}^{-1}$ may had been partially truncated by the laser filter. 


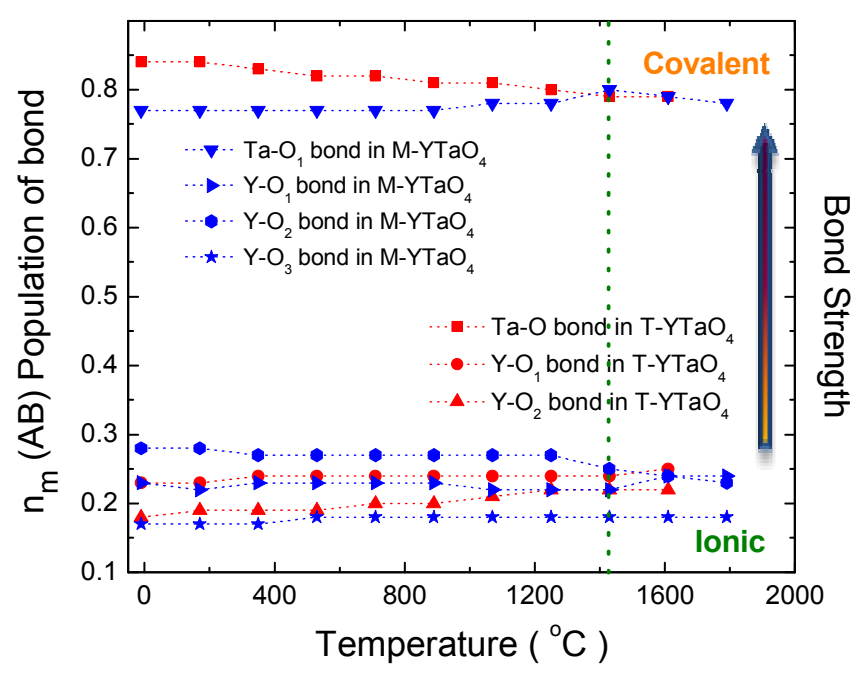

Fig 11. Population of the cation-oxygen bonds as a function of temperature for the tetragonal and monoclinic phases. The Ta-O and $\mathrm{Y}-\mathrm{O}$ bonds have covalent and ionic characteristics, respectively, at all temperatures. 


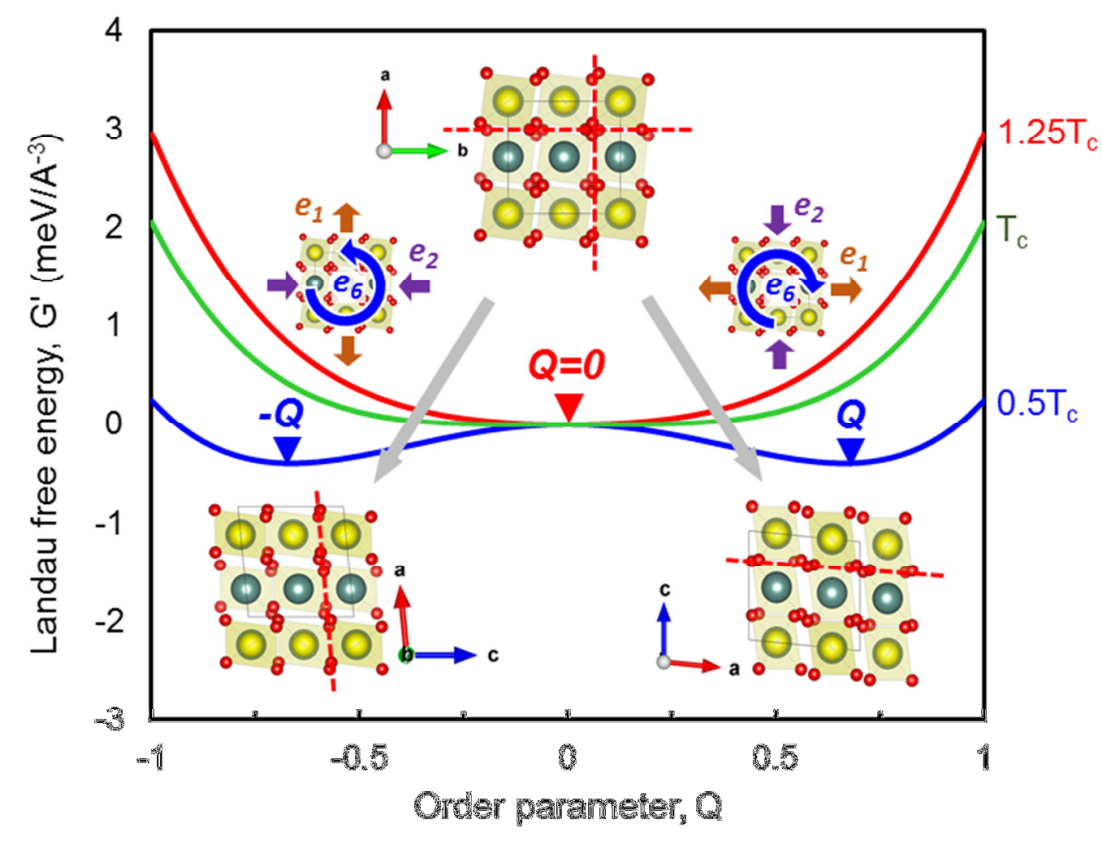

Fig 12. The Landau free energy, $G^{\prime}$, as a function of order parameter, $Q$, plotted at different temperatures. The relation of the two monoclinic variant relative to the parent tetragonal phases and associated symmetry breaking strains are also shown. Note the location of the unit cell and the orientation of closedpacked plane of oxygen (shown as dashed lines) in each monoclinic variants with respect to the parent tetragonal axis. 


\section{Tables}

Table 1 Lattice parameters and atomic positions of monoclinic and tetragonal $\mathrm{YTaO}_{4}$ in ground state by experiments (M- is at room temperature and T- is at $1475^{\circ} \mathrm{C}$ ) and first-principles calculations (at $\left.0 \mathrm{~K}\right)$. M$\mathrm{YTaO}_{4}$ is $\mathrm{C}_{2} / \mathrm{c}$ space group (15) where $\mathrm{Y}$, Ta atoms are both in the 4e Wyckoff position, and $\mathrm{O}$ atoms are in the $8 \mathrm{f}$ Wyckoff position. $\mathrm{T}-\mathrm{YTaO}_{4}$ is $\mathrm{I}_{41} / \mathrm{a}$ space group (88), where $\mathrm{Y}$, Ta, and $\mathrm{O}$ atoms are in the $4 \mathrm{~b}, 4 \mathrm{a}$, and $16 f$ Wyckoff position, respectively.

\begin{tabular}{|c|c|c|c|c|c|c|c|c|c|}
\hline \multirow{2}{*}{ Phase } & & \multirow{2}{*}{$\begin{array}{l}a \\
(\AA)\end{array}$} & \multirow{2}{*}{$\begin{array}{l}b \\
(\AA)\end{array}$} & \multirow{2}{*}{$\begin{array}{l}c \\
(\AA)\end{array}$} & \multirow{2}{*}{$\begin{array}{l}\beta^{\prime} \\
\left({ }^{\circ}\right)\end{array}$} & \multirow{2}{*}{$\begin{array}{c}\mathrm{V} \\
\left(\AA^{3}\right)\end{array}$} & \multicolumn{3}{|c|}{ Atom positions } \\
\hline & & & & & & & $\mathrm{Y}$ & $\mathrm{Ta}$ & $\mathrm{O}$ \\
\hline \multirow{3}{*}{ M } & exp. ${ }^{a}$ & 5.2393 & 10.8939 & 5.0563 & 95.52 & 287.2 & $(0.25,0.622,0)$ & $(0.25,0.145,0)$ & $\begin{array}{l}(0.090,0.461,0.252) \\
(-0.003,0.717,0.290)\end{array}$ \\
\hline & exp. & 5.3253 & 10.9283 & 5.0525 & 95.5 & 292.7 & $(0.6175,0,0.5)$ & $(0.1485,0,0.5)$ & $\begin{array}{l}(0.0895,0.45545,0.24453) \\
(-0.02967,0.71743,0.27772)\end{array}$ \\
\hline & cal. & 5.1923 & 10.6639 & 4.9299 & 95.46 & 272.16 & $(0.25,0.620,0)$ & $(0.25,0.147,0)$ & $\begin{array}{l}(0.091,0.462,0.259) \\
(-0.0143,0.722,0.286)\end{array}$ \\
\hline \multirow[t]{2}{*}{$\mathrm{T}$} & exp. & 5.2521 & & 11.15993 & & 307.84 & $(0,0,0.5)$ & $(0,0,0)$ & $(0.20096,-0.14672,0.07911)$ \\
\hline & cal. & 5.0985 & & 10.8129 & & 281.08 & $(0,0,0.6)$ & $(0,0,1)$ & $(0.24075,-0.18263,0.09334)$ \\
\hline
\end{tabular}

a: exp in ref. ICSD\# 109910. 
Table 2 Elastic constants $(\mathrm{GPa})$ of monoclinic and tetragonal $\mathrm{YTaO}_{4}$ calculated by first-principles at $0 \mathrm{~K}$

\begin{tabular}{|c|c|c|c|c|c|c|c|c|c|c|c|c|c|c|c|c|c|}
\hline phase & $\mathrm{c}_{11}{ }^{0}$ & $\mathrm{c}_{22}{ }^{0}$ & $\mathrm{c}_{33}{ }^{\circ}$ & $\mathrm{c}_{44}{ }^{0}$ & $\mathrm{c}_{55}{ }^{0}$ & $\mathrm{c}_{66}{ }^{0}$ & $\mathrm{c}_{12}{ }^{0}$ & $\mathrm{c}_{13}{ }^{0}$ & $\mathrm{c}_{15}{ }^{\mathrm{J}}$ & $\mathrm{c}_{23}{ }^{0}$ & $\mathrm{c}_{25}{ }^{\circ}$ & $\mathrm{c}_{35}{ }^{\circ}$ & $\mathrm{c}_{46}{ }^{0}$ & $\mathrm{c}_{16}{ }^{0}$ & B & $\mathrm{G}$ & $E$ \\
\hline $\mathrm{M}$ & 354.9 & 291.9 & 343 & 88.1 & 82.8 & 73.5 & 112.3 & 146.1 & 1.0 & 122.5 & -20.3 & -57.5 & -1.6 & & 183.7 & 63.2 & 170.1 \\
\hline $\mathrm{T}$ & 242.6 & & 183.3 & 29.3 & & 25.4 & 117.7 & 70.3 & & & & & & 31.8 & 128.9 & 52.7 & 139.1 \\
\hline
\end{tabular}


Table 3 Parameters used in Landau theory for tetragonal to monoclinic $\mathrm{YTaO}_{4}$ phase transition

\begin{tabular}{lccccccc}
\hline parameter & $\mathrm{a}^{\prime}$ & $\mathrm{b}^{\prime}$ & $\mathrm{b}^{*}$ & $\lambda_{1}$ & $\lambda_{2}$ & $\lambda_{3}$ & $\lambda_{4}$ \\
\hline value & 0.004243 & 8.2854 & 8.2155 & 12.272 & 13.8325 & -0.00015 & 0.000454 \\
\hline
\end{tabular}


Table 4 The experimental (at room temperature) and the first-principles calculated (at $0 \mathrm{~K}$ ) frequencies of Raman modes are shown for both monoclinic and tetragonal $\mathrm{YTaO}_{4}$.

\begin{tabular}{|c|c|c|c|c|c|}
\hline \multicolumn{4}{|c|}{$\mathrm{M}^{-\mathrm{YTaO}_{4}}$} & \multicolumn{2}{|c|}{ T-YTaO 4} \\
\hline \multirow{2}{*}{ Modes } & \multicolumn{2}{|c|}{ experiment } & \multirow{2}{*}{$\begin{array}{l}\text { cal. } \\
\left(\mathrm{cm}^{-1}\right)\end{array}$} & \multirow{2}{*}{ Modes $^{b}$} & \multirow{2}{*}{$\begin{array}{l}\text { cal. } \\
\left(\mathrm{cm}^{-1}\right)\end{array}$} \\
\hline & $\left(\mathrm{cm}^{-1}\right)$ & Intensity & & & \\
\hline $\mathrm{B}_{\mathrm{g}}$ & $119^{\mathrm{a}}$ & strong & 109 & $\mathrm{~T}\left(\mathrm{E}_{\mathrm{g}}\right)$ & 179 \\
\hline $\mathrm{A}_{\mathrm{g}}$ & 127 & weak & 133 & $\mathrm{~T}\left(\mathrm{~B}_{\mathrm{g}}\right)$ & 181 \\
\hline $\mathrm{B}_{\mathrm{g}}$ & 160 & weak & 171 & $\mathrm{~T}\left(\mathrm{~B}_{\mathrm{g}}\right)$ & 203 \\
\hline $\mathrm{A}_{\mathrm{g}}$ & 201 & medium & 207 & $\mathrm{~T}\left(\mathrm{E}_{\mathrm{g}}\right)$ & 257 \\
\hline $\mathrm{B}_{\mathrm{g}}$ & 223 & medium & 238 & $\mathrm{R}\left(\mathrm{A}_{\mathrm{g}}\right)$ & 298 \\
\hline $\mathrm{B}_{\mathrm{g}}$ & 241 & medium & 251 & $R\left(E_{g}\right)$ & 309 \\
\hline $\mathrm{A}_{\mathrm{g}}$ & 327 & strong & 307 & $\mathrm{v} 2\left(\mathrm{~A}_{\mathrm{g}}\right)$ & 393 \\
\hline $\mathrm{A}_{\mathrm{g}}$ & 349 & strong & 346 & $\mathrm{v} 2\left(\mathrm{~B}_{\mathrm{g}}\right)$ & 442 \\
\hline $\mathrm{B}_{\mathrm{g}}$ & 378 & weak & 367 & $\mathrm{v} 4\left(\mathrm{~B}_{\mathrm{g}}\right)$ & 514 \\
\hline $\mathrm{A}_{\mathrm{g}}$ & - & & 405 & $\mathrm{v} 4\left(\mathrm{E}_{\mathrm{g}}\right)$ & 591 \\
\hline $\mathrm{B}_{\mathrm{g}}$ & 427 & weak & 421 & v3( $\left.E_{g}\right)$ & 625 \\
\hline $\mathrm{B}_{\mathrm{g}}$ & 457 & weak & 466 & $\mathrm{v} 3\left(\mathrm{~B}_{\mathrm{g}}\right)$ & 749 \\
\hline $\mathrm{B}_{\mathrm{g}}$ & 487 & weak & 522 & $\operatorname{vl}\left(A_{g}\right)$ & \\
\hline \multirow[t]{3}{*}{$\mathrm{A}_{g}$} & 531 & very weak & 529 & & \\
\hline & 671 & medium & - & & \\
\hline & 705 & weak & - & & \\
\hline $\mathrm{A}_{\mathrm{g}}$ & 825 & strong & 824 & & \\
\hline $\mathrm{B}_{\mathrm{g}}$ & - & & 831 & & \\
\hline $\mathrm{B}_{\mathrm{g}}$ & - & & 901 & & \\
\hline $\mathrm{A}_{\mathrm{g}}$ & - & & 995 & & \\
\hline
\end{tabular}

a: Maybe cut off by laser filter;

b: Ref. by F. J. Manjon ${ }^{49}$. 
Table 5 Comparison of the vibrational modes of compositionally related Fergusonite $\mathrm{ABO}_{4}$ compounds.

\begin{tabular}{lllll}
\hline Energy range & $\mathrm{YTaO}_{4}$ & $\mathrm{YbTaO}_{4}$ & $\mathrm{YVO}_{4}$ & $\mathrm{YNbO}_{4}$ \\
\hline Low & $\mathrm{E}_{\mathrm{u}}$ & $\mathrm{A}_{\mathrm{u}}$ & $\mathrm{B}_{\mathrm{g}}$ & $\mathrm{B}_{\mathrm{g}}$ \\
Low & $\mathrm{A}_{\mathrm{u}}$ & $\mathrm{E}_{\mathrm{u}}$ & $\mathrm{E}_{\mathrm{g}}$ & $\mathrm{E}_{\mathrm{u}}$ \\
Medium & $\mathrm{B}_{\mathrm{g}}$ & $\mathrm{B}_{\mathrm{g}}$ & $\mathrm{E}_{\mathrm{u}}$ & $\mathrm{A}_{\mathrm{u}}$ \\
Medium & $\mathrm{E}_{\mathrm{g}}$ & $\mathrm{E}_{\mathrm{g}}$ & $\mathrm{A}_{\mathrm{u}}$ & $\mathrm{E}_{\mathrm{g}}$ \\
High & $\mathrm{A}_{\mathrm{g}}$ & $\mathrm{A}_{\mathrm{g}}$ & $\mathrm{A}_{\mathrm{g}}$ & $\mathrm{A}_{\mathrm{g}}$ \\
High & $\mathrm{B}_{\mathrm{u}}$ & $\mathrm{B}_{\mathrm{u}}$ & $\mathrm{B}_{\mathrm{u}}$ & $\mathrm{B}_{\mathrm{u}}$ \\
\hline
\end{tabular}

